Journal of Scientific Computing manuscript No.

(will be inserted by the editor)

\title{
Simulation of Dynamic Earthquake Ruptures in Complex Geometries Using High-Order Finite Difference Methods
}

\author{
Jeremy E. Kozdon · Eric M. Dunham • Jan \\ Nordström
}

Received: date / Accepted: date

Submitted to the Journal of Scientific Computing on 15 September 2011

Abstract We develop a stable and high-order accurate finite difference method for problems in earthquake rupture dynamics capable of handling complex geometries and multiple faults. The bulk material is an isotropic elastic solid cut by preexisting fault interfaces. The fields across the interfaces are related through friction laws which depend on the sliding velocity, tractions acting on the interface, and state variables which evolve according to ordinary differential equations involving local fields.

The method is based on summation-by-parts finite difference operators with irregular geometries handled through coordinate transforms and multi-block meshes. Boundary conditions as well as block interface conditions (whether frictional or otherwise) are enforced weakly through the simultaneous approximation term method, resulting in a provably stable discretization.

The theoretical accuracy and stability results are confirmed with the method of manufactured solutions. The practical benefits of the new methodology are illustrated in a simulation of a subduction zone megathrust earthquake, a challenging application problem involving complex free-surface topography, nonplanar faults, and varying material properties.

Keywords high-order finite difference · nonlinear boundary conditions .

simultaneous approximation term method · elastodynamics · summation-by-

J. E. Kozdon

Department of Geophysics, Stanford University, Stanford, CA, USA

650-723-0773 (tel)

650-725-7344 (fax)

E-mail: jkozdon@stanford.edu

E. M. Dunham

Department of Geophysics and Institute for Computational and Mathematical Engineering, Stanford University, Stanford, CA, USA

J. Nordström

Department of Mathematics, Linköping University, Linköping, Sweden 
parts · friction · wave propagation · multi-block · coordinate transforms · weak boundary conditions

\section{Introduction}

In this paper we develop a numerical method that can be applied to problems of propagating shear ruptures, particularly those arising in earthquake rupture dynamics. The rupture problem couples seismic wave propagation and frictional sliding on faults, and is thus influenced by both fault and surrounding material response. Over the temporal and spatial scales of interest in rupture dynamics, the rock response can be approximated as linear elastic (though more complex models can be considered which account for inelastic deformation) and the fault as an infinitesimally thin frictional interface.

As the material on one side of the fault is displaced with respect to the other, jump conditions relate stress and velocity on the two sides of the fault. The tangential displacement discontinuity across the interface is referred to as slip. The jump conditions arise from force balance considerations and constitutive laws for evolving fault strength (i.e., friction laws). Friction laws used in rupture dynamics, which are described in more detail below, depend not only on the current slip velocity and tractions acting on the fault but also on, for instance, the history of sliding, frictional heat generation, and transport of heat and pore fluids in the fault zone. The latter processes are parameterized in terms of "state variables" that obey differential evolution equations which are distinct from the governing equations of the elastic medium.

Earthquake simulations, and other frictional contact problems, are inherently interface driven. Hence numerical errors and instabilities arising from the treatment of the frictional interfaces can destroy the accuracy of the solution, preventing reliable ground motion predictions that are critical for seismic hazard assessment.

\subsection{Rate-and-State Friction}

A widely used mathematical framework known as rate-and-state friction captures many features observed in laboratory friction experiments (Dieterich, 1979; Rice, 1983; Ruina, 1983; Rice and Ruina, 1983; Marone, 1998; Rice et al., 2001). In its simplest form, the fault shear strength $\tau$ (resistance to slip) is

$$
\tau=\sigma_{n} f(V, \psi)
$$

where $\sigma_{n}$ is the normal stress acting on the fault (taken to be positive in compression), and the friction coefficient $f$ depends on the slip velocity, $V$, and a single state variable, $\psi$, that obeys the evolution law

$$
\frac{d \psi}{d t}=G(V, \psi)
$$

Under conditions of steady sliding at constant velocity $V$, the state variable evolves toward a steady state value $\psi_{s s}(V)$, defined implicitly through

$$
0=G\left(V, \psi_{s s}(V)\right) \text {. }
$$




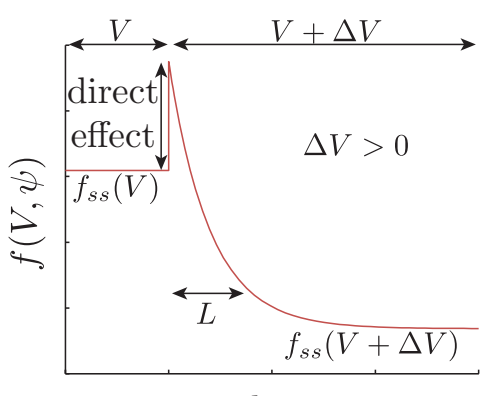

slip

Fig. 1: Response of the friction coefficient, $f$, to an abrupt increase in slip velocity from $V$ to $V+\Delta V$, for a friction law that is instantaneously velocity-strengthening $(\partial f(V, \psi) / \partial V>0)$ with a velocity-weakening steady state $\left(d f_{s s}(V) / d V<0\right) . f$ instantaneously increases from the steady state value $f_{s s}(V)$ (the direct effect), then evolves with slip over the state evolution distance, $L$, to the new, lower steady state value $f_{s s}(V+\Delta V)$.

Similarly, under steady sliding at velocity $V$, the friction coefficient approaches its steady state value,

$$
f_{s s}(V)=f\left(V, \psi_{s s}(V)\right) .
$$

A widely used state evolution law is the slip law (Rice, 1983):

$$
G(V, \psi)=-\frac{V}{L}\left[f(V, \psi)-f_{s s}(V)\right],
$$

which describes evolution of $f$ toward $f_{s s}(V)$ with slip over the state evolution distance $L$.

The steady state velocity dependence may be either velocity-strengthening $\left(f_{s s}^{\prime}(V)>0\right)$ or velocity-weakening $\left(f_{s s}^{\prime}(V)<0\right)$. Velocity-weakening interfaces between prestressed elastic solids are prone to instability. This is because the release of strain energy from the elastic medium in response to slip drives the interface to faster slip rates, which further weakens the interface and causes even more rapid sliding. This runaway weakening process is widely regarded as the cause of earthquakes.

Experiments in which a sudden change from steady sliding at velocity $V$ to $V+\Delta V$ is imposed (velocity stepping tests) reveal an even richer behavior, as illustrated in Figure 1. Rather than abruptly changing from $f_{s s}(V)$ to $f_{s s}(V+\Delta V)$, $f$ first jumps to a new value (generally different from $f_{s s}(V+\Delta V)$ ) and then evolves with slip to the new steady state $f_{s s}(V+\Delta V)$. The instantaneous change in $f$, known as the direct effect, always makes the initial response velocity-strengthening $(\partial f / \partial V>0)$. This stabilizes the fault over short time scales, which translates to a stabilization against short wavelength perturbations for sliding between elastic solids (Rice and Ruina, 1983; Rice et al., 2001). 


\subsection{Related Work on Seismic Wave Propagation and Dynamic Rupture Modeling}

Before presenting our approach, we briefly review previous work on earthquake rupture modeling. The boundary integral equation method (BIEM) has been quite popular for crack and rupture propagation problems (Das, 1980; Andrews, 1985; Das and Kostrov, 1988; Perrin et al., 1995; Geubelle and Rice, 1995; Kame and Yamashita, 1999; Aochi et al., 2000; Lapusta et al., 2000; Day et al., 2005; Noda et al., 2009). BIEM reduces the problem to solving only for the solution along the fault, with the material response entering through convolutions over the past history of slip or tractions on the fault. The main computational cost is associated with the convolutions. At least in present formulations, BIEM is limited to faults in a linear elastic medium with uniform material properties.

Finite element methods (FEM) overcome some of these computational challenges, and have been successfully applied to rupture problems (Oglesby et al., 1998; Aagaard et al., 2001; Ma and Liu, 2006; Moczo et al., 2007). In contrast to BIEM, the entire volume is discretized, not just the faults. The meshes for FEM can be quite general and typically lower order elements with linear basis functions are used. Traditional FEM discretizations of the elastic wave equation result in nondiagonal mass matrices. Solving the resulting linear system can be avoided by lumping the mass matrix. This makes the spatial difference operator rank-deficient, leading to nonphysical oscillations (hourglass modes) that must be damped. To address this the use of certain high-order methods, such as spectral element methods (Ampuero, 2002; Festa and Vilotte, 2005; Kaneko et al., 2008), which have diagonal mass matrices due to the choice of basis functions and quadrature points, and discontinuous Galerkin methods (de la Puente et al., 2009), which have block-diagonal mass matrices, are gaining in popularity.

Finite difference methods, especially those on staggered grids, are also commonly used (Andrews, 1976; Miyatake, 1980; Day, 1982; Madariaga et al., 1998; Day et al., 2005; Moczo et al., 2007). These methods are very efficient for wave propagation, but proving stability is difficult with nonlinear friction laws (Rojas et al., 2009). Additionally, staggered grid methods have difficulty handling complex geometries. A notable exception is the approach of Cruz-Atienza and Virieux (2004) for nonplanar faults.

Within seismic wave propagation there has been work on the use of unstaggered grids for the first and second order form of the equations of elasticity (Bayliss et al., 1986; Zhang and Chen, 2009; Nilsson et al., 2007). Coordinate transforms have been applied to both forms of the equations to handle complex geometries (Fornberg, 1988; Tessmer et al., 1992; Appelö and Petersson, 2009).

\subsection{Computational Approach}

This work is related to our previous paper (Kozdon et al., 2011) in which a strictly stable numerical method was developed for two dimensional antiplane shear problems with nonlinear frictional laws depending only on velocity. That study was furthermore limited to flat faults and regular (Cartesian) meshes. Here, we extend the method to tensor elasticity in irregularly shaped domains through the use of coordinate transforms. Additionally, we introduce state dependence to the friction law through the rate-and-state formalism described previously. Two of 
the main benefits of methods based on structured meshes, over unstructured mesh methods, are superior performance on parallel computers and improved dispersion properties.

As in our previous work, we use summation-by-parts (SBP) finite difference methods (Kreiss and Scherer, 1974, 1977; Strand, 1994; Carpenter et al., 1999; Mattsson and Nordström, 2004) on an unstaggered grid. An SBP difference approximation to the first derivative has the form

$$
\frac{\partial v}{\partial y} \approx \boldsymbol{H}^{-1} \boldsymbol{Q} \mathbf{v}
$$

where $\boldsymbol{H}$ is a symmetric positive definite matrix, $\boldsymbol{Q}$ is an almost skew-symmetric matrix with $\boldsymbol{Q}^{T}+\boldsymbol{Q}=\operatorname{diag}[-1,0, \ldots, 0,1]$, and the vector $\mathbf{v}=\left[\begin{array}{llll}v_{0} & v_{1} & \ldots & v_{N}\end{array}\right]^{T}$ is the grid data. These are called SBP methods because they mimic integration-byparts properties of the continuous problem. Defining the continuous and discrete norms

$$
(u, v)=\int_{a}^{b} u(y) v(y) d y \text { and }(\mathbf{u}, \mathbf{v})_{h}=\mathbf{u}^{T} \boldsymbol{H} \mathbf{v}
$$

this becomes clear since

$$
\begin{aligned}
\left(v, \frac{d v}{d y}\right) & =\int_{a}^{b} v \frac{d v}{d y} d y=\frac{1}{2}\left[v(b)^{2}-v(a)^{2}\right] \\
\left(\mathbf{v}, \boldsymbol{H}^{-1} \boldsymbol{Q} \mathbf{v}\right)_{h} & =\mathbf{v}^{T} \boldsymbol{Q} \mathbf{v}=\frac{1}{2} \mathbf{v}^{T}\left(\boldsymbol{Q}+\boldsymbol{Q}^{T}\right) \mathbf{v}=\frac{1}{2}\left(v_{N}^{2}-v_{0}^{2}\right) .
\end{aligned}
$$

SBP operators are standard central difference methods, having orders $q=$ $2,4,6,8, \ldots$ in the interior, that become one-sided methods near boundaries in a manner that ensures the SBP property. The boundary order of accuracy $r$ is typically lower than the interior accuracy $q$ and hence the global accuracy is $p=$ $r+1$ (Gustafsson, 1975; Svärd and Nordström, 2007). In this work we only consider diagonal norm (diagonal $\boldsymbol{H}$ ) operators which have interior accuracy $q=2 s(s=$ $1,2, \ldots)$, boundary accuracy $r=s$, and global accuracy $p=s+1$. There are SBP operators that have boundary accuracy $r=2 s-1$ and global accuracy $p=2 s$, but using these makes stability proofs difficult for problems with variable coefficients, coordinate transforms, and nonlinear boundary/interface conditions (Nordström and Carpenter, 2001; Olsson, 1995; Nordström, 2006; Kozdon et al., 2011).

Using SBP difference methods alone is not enough to ensure a stable method and appropriate boundary treatment is needed. Though there are many ways to incorporate boundary conditions, here we exclusively use weak enforcement of the boundary conditions through the simultaneous approximation term (SAT) method (Carpenter et al., 1994). The resulting method is provably stable, in the sense that an energy estimate exists for the semi-discrete solution (Gustafsson et al., 1996). If boundary conditions are strongly enforced (the injection method), typically stability cannot be proven, and the resulting methods are frequently unstable. This was demonstrated for frictional sliding problems by Kozdon et al. (2011).

Kozdon et al. (2011) also identified important issues regarding the stiffness of the discretization after inclusion of the nonlinear interface conditions through SAT penalty terms. Specifically, it was shown that unless the penalty terms were expressed in terms of the characteristic variables, the resulting system of equations 
can become arbitrarily stiff, meaning that efficient explicit time integration is not possible for certain portions of the parameter space.

The remainder of the paper is as follows: In Sec. 2 the continuous problem involving tensor elasticity, coordinate transforms, and nonlinear friction laws is introduced and an energy estimate is established. A stable discretization of this problem is derived in Sec. 3 using SBP difference operators and the SAT method for incorporating boundary and fault conditions. Numerical examples confirm the theoretical results in Sec. 4 and conclusions are drawn in Sec. 5.

\section{Continuous Problem}

Consider a 2-D elastic medium with a frictional fault defined by the curve $\eta(x, y)=$ 0 (Figure 2). Before discussing the frictional properties of the fault we outline the equations governing the linear elastic medium. Momentum conservation is

$$
\rho \frac{\partial v_{i}}{\partial t}=\frac{\partial \sigma_{i j}}{\partial x_{j}}, i=x, y, z,
$$

where here, as in subsequent equations, summation is implied over $x, y, z$ for repeated (Roman) subscript indices. $\rho$ is the density, $v_{i}$ are components of the particle velocity vector, and $\sigma_{i j}=\sigma_{j i}$ are components of the stress tensor. Hooke's law for isotropic elasticity is

$$
\frac{\partial \sigma_{i j}}{\partial t}=\lambda \delta_{i j} \frac{\partial v_{k}}{\partial x_{k}}+G\left(\frac{\partial v_{i}}{\partial x_{j}}+\frac{\partial v_{j}}{\partial x_{i}}\right), i, j=x, y, z,
$$

where $G$ is the shear modulus, $\lambda$ is Láme's first parameter, and $\delta_{i j}$ is the Kronecker delta. Additionally, in order for the problem to be hyperbolic (and well-posed) $\rho>0, G \geq 0$, and $\lambda+2 G / 3 \geq 0$. It is well known that (10) and (11) admit three wave types: two transversely polarized waves, known as shear or $\mathrm{S}$ waves, with wave speeds $c_{s}=\sqrt{G / \rho}$, and a single longitudinally polarized wave, known as the dilatational or $\mathrm{P}$ wave, with wave speed $c_{p}=\sqrt{(\lambda+2 G) / \rho}$. Since we are considering the 2 -D problem, the solution is invariant in the $z$-direction and in (10) and (11), derivatives with respect to $z$ are zero.

The first order hyperbolic system of equations (10) and (11) can be symmetrized by introducing the change of variables:

$$
\frac{\partial \mathbf{q}}{\partial t}=\boldsymbol{A}_{i} \frac{\partial \mathbf{q}}{\partial x_{i}}, \mathbf{q}=\frac{1}{\sqrt{2 \rho}}\left[\begin{array}{c}
\rho v_{x} \\
\rho v_{y} \\
\rho v_{z} \\
s_{x x} /\left(\sqrt{2} c_{s}\right)+\sigma_{m} / \sqrt{3 c_{p}^{2}-4 c_{s}^{2}} \\
s_{y y} /\left(\sqrt{2} c_{s}\right)+\sigma_{m} / \sqrt{3 c_{p}^{2}-4 c_{s}^{2}} \\
s_{z z} /\left(\sqrt{2} c_{s}\right)+\sigma_{m} / \sqrt{3 c_{p}^{2}-4 c_{s}^{2}} \\
\sigma_{y x} / c_{s} \\
\sigma_{x z} / c_{s} \\
\sigma_{y z} / c_{s}
\end{array}\right]
$$


for the mean stress $\sigma_{m}=\frac{1}{3}\left(\sigma_{x x}+\sigma_{y y}+\sigma_{z z}\right)$, the components of the deviatoric stress tensor $s_{i j}=\sigma_{i j}-\sigma_{m} \delta_{i j}$. The matrices in (12) are

$$
\begin{aligned}
\boldsymbol{A}_{x} & =\left[\begin{array}{ccccccccc}
0 & 0 & 0 & a_{2} & a_{1} & a_{1} & 0 & 0 & 0 \\
0 & 0 & 0 & 0 & 0 & 0 & c_{s} & 0 & 0 \\
0 & 0 & 0 & 0 & 0 & 0 & 0 & c_{s} & 0 \\
a_{2} & 0 & 0 & 0 & 0 & 0 & 0 & 0 & 0 \\
a_{1} & 0 & 0 & 0 & 0 & 0 & 0 & 0 & 0 \\
a_{1} & 0 & 0 & 0 & 0 & 0 & 0 & 0 & 0 \\
0 & c_{s} & 0 & 0 & 0 & 0 & 0 & 0 & 0 \\
0 & 0 & c_{s} & 0 & 0 & 0 & 0 & 0 & 0 \\
0 & 0 & 0 & 0 & 0 & 0 & 0 & 0 & 0
\end{array}\right], \boldsymbol{A}_{y}=\left[\begin{array}{ccccccccc}
0 & 0 & 0 & 0 & 0 & 0 & c_{s} & 0 & 0 \\
0 & 0 & 0 & a_{1} & a_{2} & a_{1} & 0 & 0 & 0 \\
0 & 0 & 0 & 0 & 0 & 0 & 0 & 0 & c_{s} \\
0 & a_{1} & 0 & 0 & 0 & 0 & 0 & 0 & 0 \\
0 & a_{2} & 0 & 0 & 0 & 0 & 0 & 0 & 0 \\
0 & a_{1} & 0 & 0 & 0 & 0 & 0 & 0 & 0 \\
c_{s} & 0 & 0 & 0 & 0 & 0 & 0 & 0 & 0 \\
0 & 0 & 0 & 0 & 0 & 0 & 0 & 0 & 0 \\
0 & 0 & c_{s} & 0 & 0 & 0 & 0 & 0 & 0
\end{array}\right], \\
a_{1} & =\frac{1}{3}\left(\sqrt{3 c_{p}^{2}-4 c_{s}^{2}}-\sqrt{2} c_{s}\right), a_{2}=\frac{1}{3}\left(\sqrt{3 c_{p}^{2}-4 c_{s}^{2}}+2 \sqrt{2} c_{s}\right),
\end{aligned}
$$

where $\boldsymbol{A}_{z} \partial \mathbf{q} / \partial z=\mathbf{0}$ since we only consider the 2-D problem. Note that $3 c_{p}^{2}-4 c_{s}^{2}>0$ if $\lambda+2 G / 3, G, \rho>0$, and that $a_{1}$ and $a_{2}$ are real. By using the symmetrized equations the analysis that follows is simplified, but the developed methods may be implemented with the original variable set. The form of the symmetrized equations is not unique, but this symmetrization results in a norm

$$
\begin{aligned}
\|\mathbf{q}(\cdot, t)\|^{2} & =\iint_{\Omega} \mathbf{q}^{T} \mathbf{q} d x d y \\
& =\iint_{\Omega}\left[\frac{\rho}{2} v_{i} v_{i}+\frac{1}{4 G} \sigma_{i j}\left(\sigma_{i j}-\frac{\lambda}{3 \lambda+2 G} \sigma_{k k} \delta_{i j}\right)\right] d x d y,
\end{aligned}
$$

where $\Omega$ is the domain of the problem, corresponding to the total mechanical energy per unit distance in the $z$-direction (Slaughter, 2002). In (15) the first term is the material kinetic energy and the second term is the elastic strain energy, and both terms are always non-negative.

Given an irregular physical domain $\Omega$ we transform to a regular computational domain $\tilde{\Omega}=[-1,1] \times[-1,1]$ (see Figure 2) using the transforms $x=x(\xi, \eta)$ and $y=y(\xi, \eta)$ along with the inverse transforms $\xi=\xi(x, y)$ and $\eta=\eta(x, y)$. We assume that $-1 \leq \xi, \eta \leq 1$ and that the fault is located at $\eta=0$. The Jacobian determinant is

$$
J=\frac{\partial x}{\partial \xi} \frac{\partial y}{\partial \eta}-\frac{\partial y}{\partial \xi} \frac{\partial x}{\partial \eta}
$$

which gives rise to the metric relations

$$
J \frac{\partial \xi}{\partial x}=\frac{\partial y}{\partial \eta}, J \frac{\partial \xi}{\partial y}=-\frac{\partial x}{\partial \eta}, J \frac{\partial \eta}{\partial y}=\frac{\partial x}{\partial \xi}, J \frac{\partial \eta}{\partial x}=-\frac{\partial y}{\partial \xi} .
$$

In the transformed coordinate system, the governing equations (12) are typically written in conservation form, but in order to derive a provably stable numerical method we instead write the more general formula (Nordström, 2006)

$$
\begin{aligned}
J \frac{\partial \mathbf{q}}{\partial t} & =(1-\gamma)\left(\frac{\partial \mathcal{F}_{\xi}}{\partial \xi}+\frac{\partial \mathcal{F}_{\eta}}{\partial \eta}\right)+\gamma J\left(\frac{\partial \xi}{\partial x_{i}} \boldsymbol{A}_{i} \frac{\partial \mathbf{q}}{\partial \xi}+\frac{\partial \eta}{\partial x_{i}} \boldsymbol{A}_{i} \frac{\partial \mathbf{q}}{\partial \eta}\right) \\
\mathcal{F}_{\xi} & =J \frac{\partial \xi}{\partial x_{i}} \boldsymbol{A}_{i} \mathbf{q}, \quad \mathcal{F}_{\eta}=J \frac{\partial \eta}{\partial x_{i}} \boldsymbol{A}_{i} \mathbf{q}
\end{aligned}
$$




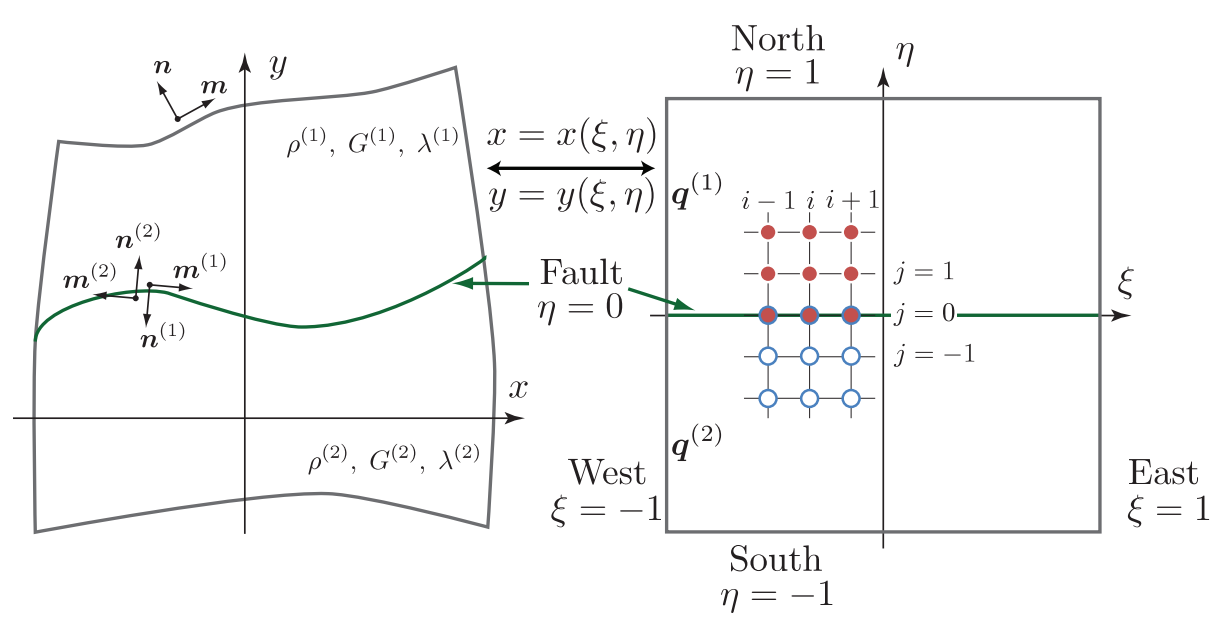

Fig. 2: Problem configuration and computational domain in both the original and transformed coordinate system. In the new coordinate system the grid is regular. Material properties and solution for $\eta>0$ are denoted with a superscript (1); those for $\eta<0$ are denoted with (2). $\mathbf{n}$ in the unit outward normal along the block boundaries, and the unit vector $\mathbf{m}$ is orthogonal to the unit normal such that $\mathbf{n} \times \mathbf{m}=\hat{\mathbf{z}}$. The exterior boundaries are referred to as north, south, east, and west as shown.

with $\gamma \in[0,1]$. If $\gamma=0$ the equations are in conservative form. Note that the symmetric part of the governing equations is not included in (18) as it is zero due to the metric relations and the chain rule:

$$
\gamma\left[\frac{\partial}{\partial \xi}\left(J \frac{\partial \xi}{\partial x_{i}}\right)+\frac{\partial}{\partial \eta}\left(J \frac{\partial \eta}{\partial x_{i}}\right)\right] \boldsymbol{A}_{i} \mathbf{q}=\mathbf{0}
$$

In the transformed domain, energy in the solution (15) becomes

$$
\begin{aligned}
\|\mathbf{q}(\cdot, t)\|^{2} & =\iint_{\tilde{\Omega}} \mathbf{q}^{T} J \mathbf{q} d \xi d \eta \\
& =\iint_{\tilde{\Omega}}\left[\frac{1}{2} \rho v_{i} v_{i}+\frac{1}{4 G} \sigma_{i j}\left(\sigma_{i j}-\frac{\lambda}{3 \lambda+2 G} \sigma_{k k} \delta_{i j}\right)\right] J d \xi d \eta
\end{aligned}
$$

After the inclusion of boundary and interface conditions we use the following definition of well-posedness (Gustafsson et al., 1996):

Definition 1 The governing equations (18) with homogenous boundary conditions are said to be well-posed if a unique solution exists and the estimate

$$
\|\mathbf{q}(\cdot, t)\| \leq K_{c} e^{\alpha_{c} t}\|\mathbf{q}(\cdot, 0)\|
$$

holds for constants $K_{c}$ and $\alpha_{c}$ independent of the solution. 
We refer to (22) as the energy estimate as it bounds the future energy of the solution in terms of the initial condition. Furthermore, if we can show that the energy dissipation rate is negative semidefinite, i.e.,

$$
\frac{d}{d t}\|\mathbf{q}(\cdot, t)\| \leq 0
$$

then the energy estimate (22) follows by integration with $K_{c}=1$ and $\alpha_{c}=0$.

\subsection{Tractions, Velocities, and Characteristic Variables}

In order to define the relations that govern the exterior boundaries and fault we need to define a few physical concepts. Consider the unit normal to a surface $\mathbf{n}$ (in the original coordinate system) along with orthonormal vectors $\hat{\mathbf{z}}$ (the unit vector in the $z$-direction) and $\mathbf{m}=\left[n_{y}-n_{x} 0\right]^{T}$, i.e., $\mathbf{n} \times \mathbf{m}=\hat{\mathbf{z}}$. The tractions (or forces per unit area) on this surface are $T_{i}=\sigma_{i j} n_{j}$. The normal stress on the surface is $\sigma_{n}=-n_{i} T_{i}=-n_{i} \sigma_{i j} n_{j}$, where the minus sign is due to the convention of positive normal stress in compression. The shear traction vector is

$$
\tau_{i}=\left(\delta_{i j}-n_{i} n_{j}\right) T_{j}=\tau_{m} m_{i}+\tau_{z} \hat{z}_{i}
$$

and $\tau_{m}=m_{j} \tau_{j}$ is the magnitude of $\boldsymbol{\tau}$ in the $\mathbf{m}$ direction. Note that $\boldsymbol{\tau}$ is orthogonal to $\mathbf{n}$ by construction. Similarly, we define the velocity of the material in the normal direction as $v_{n i}=n_{i} n_{j} v_{j}$, with magnitude $v_{n}=v_{i} n_{i}$, and in the orthogonal direction as

$$
v_{s i}=\left(\delta_{i j}-n_{i} n_{j}\right) v_{j}=v_{m} m_{i}+v_{z} \hat{z}_{i},
$$

with $v_{m}=m_{j} v_{j}$ being the magnitude of velocity in the $\mathbf{m}$ direction.

As previously mentioned, governing equations (12) admit three wave types. The same applies to the transformed equations (18), though the propagation speeds differ and are spatially variable due to the transform. The characteristic variables associated with $\mathrm{P}$ waves propagating at speed $c_{p}$ in the $\pm \mathbf{n}$ direction are

$$
w_{P}^{ \pm}=-\sigma_{n} \mp Z_{p} v_{n}
$$

where $Z_{p}=\rho c_{p}$ is the dilatational impedance. Similarly, there are two sets of S waves propagating at speed $c_{s}$ in the $\pm \mathbf{n}$ direction, which we classify as SV and $\mathrm{SH}$ waves for polarizations in the $\mathbf{m}$ and $\hat{\mathbf{z}}$ directions, respectively. The associated characteristic variables are

$$
w_{S V}^{ \pm}=\tau_{m} \mp Z_{s} v_{m}, w_{S H}^{ \pm}=\tau_{z} \mp Z_{s} v_{z},
$$

where $Z_{s}=\rho c_{s}$ is the shear impedance. There are three additional zero speed waves in the system:

$$
\begin{aligned}
\stackrel{\circ}{\omega}_{m} & =\sigma_{i j} m_{i} m_{j}+\left(1-2 \frac{c_{s}^{2}}{c_{p}^{2}}\right) \sigma_{n}, \stackrel{\circ}{w}_{z}=\sigma_{z z}+\left(1-2 \frac{c_{s}^{2}}{c_{p}^{2}}\right) \sigma_{n}, \\
\stackrel{\circ}{w}_{m z} & =\sigma_{i z} m_{i} .
\end{aligned}
$$

For convenience we define the vectors $\mathbf{w}^{ \pm}=\left[w_{P}^{ \pm} w_{S V}^{ \pm} w_{S H}^{ \pm}\right]^{T}$ and $\stackrel{\circ}{\mathbf{w}}=\left[\stackrel{\circ}{w}_{m} \stackrel{\circ}{w}_{z} \stackrel{\circ}{w}_{m z}\right]^{T}$. 


\subsection{Boundary and Interface Conditions}

Since we have defined outward normals to the exterior boundaries (e.g., on the north boundary $\mathbf{n}=\nabla \eta /|\nabla \eta|)$, then $\mathbf{w}^{+}$is always propagating outward and $\mathbf{w}^{-}$ is always propagating inward. This implies that at each exterior boundary we need three boundary conditions relating $\mathbf{w}^{-}$to the other characteristic variables (Kreiss, 1970). In this work we assume that the exterior boundary conditions are of the linear form

$$
\mathbf{w}^{-}=\boldsymbol{R} \mathbf{w}^{+}, \boldsymbol{R}=\operatorname{diag}\left[R_{P} R_{S V} R_{S H}\right]
$$

where the reflection coefficients $\boldsymbol{R}$ can vary spatially along the boundary but are independent of the solution. Two important boundary conditions for this work are the traction-free boundary condition $\left(T_{i}=0\right)$, for which $\boldsymbol{R}=-\boldsymbol{I}$, and the nonreflecting boundary condition $\boldsymbol{R}=\mathbf{0}$. More effective nonreflecting boundary conditions are possible (e.g., Hagstrom et al., 2008; Appelö et al., 2006), but the focus of this work is interface conditions and these simple formulations are sufficient.

Across the fault, $\eta=0$, the material properties and some of the fields may be discontinuous. Notationally, we denote the fields and properties for $\eta>0$ with a superscript (1) and for $\eta<0$ we use a superscript (2) (see Figure 2). At the fault we define the two unit normal vectors (one for each side of the fault) as

$$
\mathbf{n}^{(1)}=-\frac{\nabla \eta}{|\boldsymbol{\nabla} \eta|} \quad \text { and } \quad \mathbf{n}^{(2)}=\frac{\boldsymbol{\nabla} \eta}{|\boldsymbol{\nabla} \eta|}
$$

such that the unit normal for side (1) points into side (2) and the unit normal for side (2) points into side (1). Since there are six characteristics propagating out of the fault $\left(\mathbf{w}^{-(1)}\right.$ and $\left.\mathbf{w}^{-(2)}\right)$, six interface conditions relating $\mathbf{w}^{-(l)}$ to the other characteristic variables are needed. We take these to be nonlinear relations involving all of the characteristic variables propagating into the fault,

$$
\mathbf{w}^{-(l)}=\mathcal{W}^{-(l)}\left(\mathbf{w}^{+(1)}, \mathbf{w}^{+(2)}\right) .
$$

In general $\mathcal{W}^{-(l)}$ can also be a function of the stationary characteristic variables $\stackrel{\circ}{\mathbf{w}}^{(1)}$ and $\stackrel{\circ}{\mathbf{w}}^{(2)}$, but (as will be seen below) this is not necessary for the interface conditions considered in this work.

It is more typical for the interface conditions (32) to be specified in terms of the physical variables, namely the tractions and velocities. The traction exerted on side (l) of the fault by the material on the opposite side of the fault is $T_{i}^{(l)}=\sigma_{i j}^{(l)} n_{j}^{(l)}$. Force balance requires that all components of traction be equal and opposite across the fault, that is, $T_{i}^{(1)}=-T_{i}^{(2)}$ (Slaughter, 2002) or, equivalently,

$$
\sigma_{n}^{(1)}=-\sigma_{n}^{(2)}, \boldsymbol{\tau}^{(1)}=-\boldsymbol{\tau}^{(2)}
$$

For concreteness, we define the normal stress and shear traction "on the fault" as those acting on side (2) from side (1):

$$
\sigma_{n} \equiv \sigma_{n}^{(2)} \text { and } \boldsymbol{\tau} \equiv \boldsymbol{\tau}^{(2)}
$$


Across the fault the velocities can be discontinuous. The slip velocity is the discontinuity in fault parallel velocity,

$$
V_{i}=-\sum_{l=1}^{2} v_{s i}^{(l)}=\left(\delta_{i j}-n_{i}^{(2)} n_{j}^{(2)}\right)\left(v_{j}^{(1)}-v_{j}^{(2)}\right),
$$

and the fault opening velocity is the discontinuity in fault normal velocity,

$$
\beta_{i}=-\sum_{l=1}^{2} v_{n i}^{(l)}=n_{i}^{(2)} n_{j}^{(2)}\left(v_{j}^{(1)}-v_{j}^{(2)}\right), \beta=\beta_{i} n_{i} .
$$

In this work, we assume that the fault is closed,

$$
\beta=0,
$$

and the friction law

$$
\tau=f(V, \psi) \max \left(0, \sigma_{n}\right), \quad V=\|\mathbf{V}\|_{2}, \tau=\|\boldsymbol{\tau}\|_{2},
$$

where the friction coefficient $f$ is a function of both the sliding velocity $V$ and the state variable $\psi$ (discussed further in Sec. 4.1). The $\max \left(0, \sigma_{n}\right)$ is used to avoid energy growth due to a tensile normal stress. Physically if $\sigma_{n}>0$ the normal stress is tensile and the fault surface is being pulled open, but in this model we constrain against opening $(\beta=0)$ and just set $\tau=0$ if $\sigma_{n}<0$. Additionally, we require that the slip velocity be parallel to the shear stress:

$$
\tau V_{i}=V \tau_{i}
$$

The fault conditions (38) and (39) along with the no-opening (37) and continuity conditions (33) give six relations governing the interface, this is the same number as required by the characteristic analysis. A necessary condition for the physical variable formulation to lead to a well-posed problem is the existence of a unique equivalence to the characteristic form (Kreiss, 1970). This is guaranteed by the following proposition:

Proposition 1 The fault conditions (38) and (39) along with the no-opening (37) and continuity conditions (33) can be uniquely expressed in the characteristic form (32) if

$$
\max \left(0, \sigma_{n}\right) \frac{\partial f}{\partial V} \neq-\frac{\tilde{Z}_{s}}{2}, \tilde{Z}_{s}=2\left(\frac{1}{Z_{s}^{(1)}}+\frac{1}{Z_{s}^{(2)}}\right)^{-1}
$$

The proof of Proposition 1 is given in App. A. Note that all friction laws currently used in the literature satisfy $\partial f / \partial V \geq 0$ (i.e., they are instantaneously velocitystrengthening, as discussed in the introduction and illustrated in Figure 1), thus satisfying the constraint of Proposition 1. Note also that only $\partial f(V, \psi) / \partial \psi$ must be non-negative; there are no restrictions on the sign of $d f_{s s}(V) / d V$. Therefore, subsequent weakening from state evolution (as shown in Figure 1 ) does not violate Proposition 1. 


\subsection{Energy Estimate for the Continuous Problem}

We now consider the energy dissipation properties of problem (18) with exterior boundary conditions (30) and fault conditions (33), (37), (38), and (39). Taking the time derivative of the energy (21) gives

$$
\begin{aligned}
\frac{d\|\mathbf{q}(\cdot, t)\|^{2}}{d t}= & 2 \iint_{\tilde{\Omega}} \mathbf{q}^{T} J \frac{\partial \mathbf{q}}{\partial t} d \xi d \eta \\
= & 2 \iint_{\tilde{\Omega}}\left[(1-\gamma) \mathbf{q}^{T}\left(\frac{\partial \mathcal{F}_{\xi}}{\partial \xi}+\frac{\partial \mathcal{F}_{\eta}}{\partial \eta}\right)+\gamma\left(\mathcal{F}_{\xi}^{T} \frac{\partial \mathbf{q}}{\partial \xi}+\mathcal{F}_{\eta}^{T} \frac{\partial \mathbf{q}}{\partial \eta}\right)\right] d \xi d \eta \\
= & \iint_{\tilde{\Omega}}\left[\frac{\partial}{\partial \xi}\left(\mathbf{q}^{T} \mathcal{F}_{\xi}\right)+\frac{\partial}{\partial \eta}\left(\mathbf{q}^{T} \mathcal{F}_{\eta}\right)\right] d \xi d \eta \\
& +(1-2 \gamma) \iint_{\tilde{\Omega}}\left(\mathbf{q}^{T} \frac{\partial \mathcal{F}_{\xi}}{\partial \xi}+\mathbf{q}^{T} \frac{\partial \mathcal{F}_{\eta}}{\partial \eta}-\mathcal{F}_{\xi}^{T} \frac{\partial \mathbf{q}}{\partial \xi}-\mathcal{F}_{\eta}^{T} \frac{\partial \mathbf{q}}{\partial \eta}\right) d \xi d \eta \\
= & \int_{\partial \Omega} v_{i} \sigma_{i j} n_{j} d s \\
& -\left.\int_{-1}^{1}\left(J^{(1)} \frac{\partial \eta^{(1)}}{\partial x_{i}} \sigma_{i j}^{(1)} v_{j}^{(1)}-J^{(2)} \frac{\partial \eta^{(2)}}{\partial x_{i}} \sigma_{i j}^{(2)} v_{j}^{(2)}\right)\right|_{\eta=0} d \xi
\end{aligned}
$$

where we have used the divergence theorem to change area integrals to line integrals over the exterior boundary $\partial \Omega$ and fault (the two last terms in (41), respectively) with $d s$ being the arc length along the external boundary. The area integral being multiplied by $1-2 \gamma$ is zero due to the metric relations (17) and the fact that material properties are uniform on both sides of the fault. The exterior boundary term in (41) can be rewritten using local characteristic variables:

$$
\begin{aligned}
v_{i} \sigma_{i j} n_{j}= & -v_{n} \sigma_{n}+v_{m} \tau_{m}+v_{z} \tau_{z} \\
= & \frac{1}{4 Z_{p}}\left[\left(w_{P}^{-}\right)^{2}-\left(w_{P}^{+}\right)^{2}\right]+\frac{1}{4 Z_{s}}\left[\left(w_{S H}^{-}\right)^{2}-\left(w_{S H}^{+}\right)^{2}\right] \\
& +\frac{1}{4 Z_{s}}\left[\left(w_{S V}^{-}\right)^{2}-\left(w_{S V}^{+}\right)^{2}\right] \\
= & -\frac{1}{4 Z_{p}}\left(1-R_{P}^{2}\right)\left(w_{P}^{+}\right)^{2}-\frac{1}{4 Z_{s}}\left(1-R_{S H}^{2}\right)\left(w_{S H}^{+}\right)^{2} \\
& -\frac{1}{4 Z_{s}}\left(1-R_{S V}^{2}\right)\left(w_{S V}^{+}\right)^{2}
\end{aligned}
$$

and each of the three terms in (42) are negative semidefinite if all the reflection coefficients in (30) satisfy $|R| \leq 1$. Note that $v_{i} \sigma_{i j} n_{j}=v_{i} T_{i}$ is the rate at which work (per unit surface area) is done on the interior material by tractions on the exterior boundaries (Malvern, 1977). For the fault term, first note that the metric relations (17) imply that $J^{(1)} \partial \eta^{(1)} / \partial x_{i}=J^{(2)} \partial \eta^{(2)} / \partial x_{i}$ because $\partial x_{i} / \partial \xi$ must be continuous across the fault since $x_{i}^{(1)}(\xi, 0)=x_{i}^{(2)}(\xi, 0)$. Together with the requirement that shear stress and slip velocity are parallel (39), the fault energy dissipation rate integrand in (41) becomes

$$
-J \frac{\partial \eta}{\partial x_{i}}\left(\sigma_{i j}^{(1)} v_{j}^{(1)}-\sigma_{i j}^{(2)} v_{j}^{(2)}\right) d \xi=-J|\nabla \eta|\left(-\sigma_{n} \beta+V \tau\right) d \xi
$$


which, due to the fault conditions (37) and (38), is negative semidefinite. Since $J|\nabla \eta| d \xi$ is the arc length along the fault, this integral corresponds to the total energy (per unit surface area) dissipated by work on the fault during to frictional sliding. Thus the energy dissipation rate (41) is negative semidefinite and we have an energy estimate. Note that this holds for all values of the splitting parameter $\gamma$, as must be case.

Due to the nonlinearity of the interface conditions, uniqueness of the solution does not follow directly from the energy estimate since the difference of two solutions does not satisfy the friction law (Kozdon et al., 2011). The question of well-posedness for the most general problem of sliding between dissimilar elastic materials with rate-and-state friction laws is still an open and active research question (Rice et al., 2001). Since the focus of this paper is the development of the numerical method, we do not consider this question further here.

\section{Semi-Discrete Problem}

We discretize the domain using an $\left(N_{\xi}+1\right) \times\left(N_{\eta}+1\right)$ grid on both sides of the fault with collocated grid nodes at the fault (see Figure 2). In the $\xi$-direction the grid indices are $i=0, \ldots, N_{\xi}$ and in the $\eta$-direction for $\eta \geq 0$ the grid indices are $j=0, \ldots, N_{\eta}$ and for $\eta \leq 0$ they are $j=-N_{\eta}, \ldots, 0$. Additionally, we refer to the grid node $(i, j)$ on side $(l)$ of the fault as $\left(\xi_{i}^{(l)}, \eta_{j}^{(l)}\right)=\left(i h_{\xi}-1, j h_{\eta}\right)$ where $h_{\xi}=2 / N_{\xi}$ and $h_{\eta}=1 / N_{\eta}$. By assuming that $N_{\eta}^{(1)}=N_{\eta}^{(2)}=N_{\eta}$, as we have done, the notation is greatly simplified. Of course, it is straightforward to apply the method to cases that do not satisfy this restriction. On the other hand, $N_{\xi}^{(1)}=N_{\xi}^{(2)}=N_{\xi}$ is required because the grid nodes must match along the fault.

The semi-discretization of the transformed governing equations (18) is done using dimension-by-dimension application of the 1-D SBP operators with weak enforcement of boundary and interface terms using the SAT method:

$$
\begin{aligned}
& \left(\boldsymbol{J}^{(1)} \otimes \boldsymbol{I}_{9}\right) \frac{d \mathbf{q}^{(1)}}{d t}=(1-\gamma)\left(\boldsymbol{D}_{\xi} \mathcal{F}_{\xi}^{(1)}+\boldsymbol{D}_{\eta} \mathcal{F}_{\eta}^{(1)}\right) \\
& \quad+\gamma\left(\boldsymbol{J}^{(1)} \otimes \boldsymbol{I}_{9}\right)\left[\left(\frac{\partial \boldsymbol{\xi}^{(1)}}{\partial x_{i}} \otimes \boldsymbol{A}_{i}\right) \boldsymbol{D}_{\xi}+\left(\frac{\partial \boldsymbol{\eta}^{(1)}}{\partial x_{i}} \otimes \boldsymbol{A}_{i}\right) \boldsymbol{D}_{\eta}\right] \mathbf{q}^{(1)} \\
& \quad+\boldsymbol{B} \boldsymbol{T}_{N}^{(1)}+\boldsymbol{B} \boldsymbol{T}_{E}^{(1)}+\boldsymbol{B} \boldsymbol{T}_{W}^{(1)}+\boldsymbol{F} \boldsymbol{T}^{(1)} \\
& \left(\boldsymbol{J}^{(2)} \otimes \boldsymbol{I}_{9}\right) \frac{d \mathbf{q}^{(2)}}{d t}=(1-\gamma)\left(\boldsymbol{D}_{\xi} \mathcal{F}_{\xi}^{(2)}+\boldsymbol{D}_{\eta} \mathcal{F}_{\eta}^{(2)}\right) \\
& \quad+\gamma\left(\boldsymbol{J}^{(2)} \otimes \boldsymbol{I}_{9}\right)\left[\left(\frac{\partial \boldsymbol{\xi}^{(2)}}{\partial x_{i}} \otimes \boldsymbol{A}_{i}\right) \boldsymbol{D}_{\xi}+\left(\frac{\partial \boldsymbol{\eta}^{(2)}}{\partial x_{i}} \otimes \boldsymbol{A}_{i}\right) \boldsymbol{D}_{\eta}\right] \mathbf{q}^{(2)} \\
& \quad+\boldsymbol{B} \boldsymbol{T}_{S}^{(2)}+\boldsymbol{B} \boldsymbol{T}_{E}^{(2)}+\boldsymbol{B} \boldsymbol{T}_{W}^{(2)}+\boldsymbol{F} \boldsymbol{T}^{(2)} \\
& \boldsymbol{D}_{\xi}=\boldsymbol{H}_{\xi}^{-1} \boldsymbol{Q}_{\xi} \otimes \boldsymbol{I}_{\eta} \otimes \boldsymbol{I}_{9}, \boldsymbol{D}_{\eta}=\boldsymbol{I}_{\xi} \otimes \boldsymbol{H}_{\eta}^{-1} \boldsymbol{Q}_{\eta} \otimes \boldsymbol{I}_{9},
\end{aligned}
$$

where $\boldsymbol{H}_{\xi}^{-1} \boldsymbol{Q}_{\xi}$ is the 1-D SBP operator for an $N_{\xi}+1$ grid and $\boldsymbol{H}_{\eta}^{-1} \boldsymbol{Q}_{\eta}$ for an $N_{\eta}+1$ grid, $\boldsymbol{I}_{\xi}$ and $\boldsymbol{I}_{\eta}$ are the $\left(N_{\xi}+1\right) \times\left(N_{\xi}+1\right)$ and $\left(N_{\eta}+1\right) \times\left(N_{\eta}+1\right)$ identity matrices, respectively, $\boldsymbol{I}_{9}$ is the $9 \times 9$ identity matrix, and $\otimes$ denotes the Kronecker product 
of two matrices. The grid data is "stacked" as $9\left(N_{\xi}+1\right)\left(N_{\eta}+1\right) \times 1$ vectors:

$$
\mathbf{q}^{(l)}=\left[\begin{array}{c}
\mathbf{q}_{0}^{(l)} \\
\vdots \\
\mathbf{q}_{N_{\xi}}^{(l)}
\end{array}\right], \mathbf{q}_{i}^{(1)}=\left[\begin{array}{c}
\mathbf{q}_{i 0}^{(1)} \\
\vdots \\
\mathbf{q}_{i N_{\eta}}^{(1)}
\end{array}\right], \mathbf{q}_{i}^{(2)}=\left[\begin{array}{c}
\mathbf{q}_{i,-N_{\eta}}^{(2)} \\
\vdots \\
\mathbf{q}_{i 0}^{(2)}
\end{array}\right],
$$

with $\mathbf{q}_{i j}^{(l)}(t) \approx \mathbf{q}^{(l)}\left(\xi_{i}^{(l)}, \eta_{j}^{(l)}, t\right)$. From (12) we see that the stresses and velocities are collocated. The Jacobian determinant matrices are defined as $\left(N_{\xi}+1\right)\left(N_{\eta}+1\right) \times$ $\left(N_{\xi}+1\right)\left(N_{\eta}+1\right)$ diagonal matrices:

$$
\begin{aligned}
\boldsymbol{J}^{(1)} & =\operatorname{diag}\left[\begin{array}{llll}
J_{00}^{(1)} & J_{01}^{(1)} & \cdots & J_{N_{\xi} N_{\eta}}^{(1)}
\end{array}\right] \\
\boldsymbol{J}^{(2)} & =\operatorname{diag}\left[\begin{array}{llll}
J_{0,-N_{\eta}}^{(2)} & J_{0,1-N_{\eta}}^{(2)} & \cdots & J_{N_{\xi} 0}^{(2)}
\end{array}\right],
\end{aligned}
$$

with $J_{i j}^{(l)}=J^{(l)}\left(\xi_{i}^{(l)}, \eta_{j}^{(l)}\right)$. The flux terms $\mathcal{F}$ are similarly defined as vectors

$$
\mathcal{F}_{\xi}^{(l)}=\left(\boldsymbol{J}^{(l)} \frac{\partial \boldsymbol{\xi}^{(l)}}{\partial x_{i}} \otimes \boldsymbol{A}_{i}^{(l)}\right) \mathbf{q}^{(l)}, \mathcal{F}_{\eta}^{(l)}=\left(\boldsymbol{J}^{(l)} \frac{\partial \boldsymbol{\eta}^{(l)}}{\partial x_{i}} \otimes \boldsymbol{A}_{i}^{(l)}\right) \mathbf{q}^{(l)},
$$

where $\partial \boldsymbol{\xi}^{(l)} / \partial x_{i}$ and $\partial \boldsymbol{\eta}^{(l)} / \partial x_{i}$ are diagonal matrices defined in an analogous manner to $\boldsymbol{J}^{(l)}$, and in (50) the subscript $i$ implies summation and does not refer to a grid index.

\subsection{Interface and Boundary Penalty Terms}

The fault interface terms in (44) and (45) are defined as

$$
\begin{aligned}
& \boldsymbol{F} \boldsymbol{T}^{(1)}=\left(\boldsymbol{I}_{\xi} \otimes \boldsymbol{H}_{\eta}^{-1} \boldsymbol{E}_{S} \otimes \boldsymbol{I}_{9}\right) \mathcal{P}_{F}^{(1)}, \boldsymbol{E}_{N}=\operatorname{diag}\left[\mathbf{e}_{N}\right], \mathbf{e}_{N}=\left[\begin{array}{llll}
1 & 0 & \cdots & 0
\end{array}\right]^{T}, \\
& \boldsymbol{F} \boldsymbol{T}^{(2)}=\left(\boldsymbol{I}_{\xi} \otimes \boldsymbol{H}_{\eta}^{-1} \boldsymbol{E}_{N} \otimes \boldsymbol{I}_{9}\right) \mathcal{P}_{F}^{(2)}, \boldsymbol{E}_{S}=\operatorname{diag}\left[\mathbf{e}_{S}\right], \mathbf{e}_{S}=\left[\begin{array}{llll}
0 & \cdots & 0 & 1
\end{array}\right]^{T} .
\end{aligned}
$$

The vectors $\mathcal{P}_{F}^{(1)}$ and $\mathcal{P}_{F}^{(2)}$ are defined analogously to $\mathbf{q}^{(l)}$ with all entries being zero except at the fault interface where

$$
\left(\mathcal{P}_{F}^{(l)}\right)_{i 0}=\left(\boldsymbol{\Sigma}_{F}^{(l)}\right)_{i 0}\left(\mathbf{w}_{i 0}^{-(l)}-\mathcal{W}_{i 0}^{-(l)}\right)
$$

The $9 \times 3$ penalty matrix $\left(\Sigma_{F}^{(l)}\right)_{i 0}$ will be determined below such that the method is stable. The quantities $w_{i 0}^{ \pm(l)}$ are the characteristic variables defined from $\mathbf{q}_{i 0}^{(l)}$ with respect to the outward pointing normal (see (26) and (27)) and

$$
\mathcal{W}_{i 0}^{-(l)}=\mathcal{W}^{-(l)}\left(\mathbf{w}_{i 0}^{+(1)}, \mathbf{w}_{i 0}^{+(2)}\right)
$$

as defined in (32).

The penalty terms at the boundaries are defined completely analogously, except with the nonlinear friction law replaced by the linear boundary conditions (30). For instance, the penalty term on the west boundary is

$$
\boldsymbol{B} \boldsymbol{T}_{W}^{(l)}=\left(\boldsymbol{H}_{\xi}^{-1} \boldsymbol{E}_{W} \otimes \boldsymbol{I}_{\eta} \otimes \boldsymbol{I}_{9}\right) \mathcal{P}_{W}^{(l)}, \boldsymbol{E}_{W}=\operatorname{diag}\left[\mathbf{e}_{W}\right], \mathbf{e}_{W}=\left[\begin{array}{llll}
1 & 0 & \cdots & 0
\end{array}\right]^{T},
$$


with $\mathcal{P}_{W}^{(l)}$ being a vector of zeros except at the west boundary where

$$
\left(\mathcal{P}_{W}^{(l)}\right)_{0 j}=\left(\boldsymbol{\Sigma}_{W}^{(l)}\right)_{0 j}\left(\mathbf{w}_{0 j}^{-(l)}-\boldsymbol{R}_{0 j}^{(l)} \mathbf{w}_{0 j}^{+(l)}\right) .
$$

The reflection coefficients at the boundary nodes are $\boldsymbol{R}_{0 j}^{(l)}=\boldsymbol{R}\left(\xi=-1, \eta_{j}\right)$. The other exterior boundary terms in (44) and (45) are defined similarly.

\subsection{Energy Estimate for the Semi-Discrete Problem}

The discrete energy is defined in a similar manner to the continuous energy (21):

$$
\|\mathbf{q}(t)\|_{h}^{2}=\sum_{l=1}^{2}\left(\mathbf{q}^{(l)}\right)^{T}\left(\boldsymbol{H}_{\xi} \otimes \boldsymbol{H}_{\eta} \otimes \boldsymbol{I}_{9}\right)\left(\boldsymbol{J}^{(l)} \otimes \boldsymbol{I}_{9}\right) \mathbf{q}^{(l)} .
$$

We now use the following definition for stability:

Definition 2 The semi-discrete problem (44)-(46) is said to be stable if the energy estimate

$$
\|\mathbf{q}(t)\|_{h}^{2} \leq K_{d} e^{\alpha_{d} t}\|\mathbf{q}(0)\|_{h}^{2},
$$

holds for constants $\alpha_{d}$ and $K_{d}$ independent of the initial conditions.

Multiplying the discretization (44) and (45) by $\left(\mathbf{q}^{(l)}\right)^{T}\left(\boldsymbol{H}_{\xi} \otimes \boldsymbol{H}_{\eta} \otimes \boldsymbol{I}_{9}\right)$, adding the transpose, and summing over both sides of the fault we get the energy dissipation rate

$$
\frac{d}{d t}\|\mathbf{q}(t)\|_{h}^{2}=\sum_{l=1}^{2}\left(F T^{(l)}+B T_{W}^{(l)}+B T_{E}^{(l)}+G R_{\xi}^{(l)}+G R_{\eta}^{(l)}\right)+B T_{N}^{(1)}+B T_{S}^{(2)} .
$$

The fault terms are

$$
\begin{aligned}
& F T^{(1)}=-\left(\mathbf{q}^{(1)}\right)^{T}\left(\boldsymbol{H}_{\eta} \otimes \boldsymbol{E}_{S} \otimes \boldsymbol{I}_{9}\right) \mathcal{F}_{\xi}^{(1)}+2\left(\mathbf{q}^{(1)}\right)^{T}\left(\boldsymbol{H}_{\xi} \otimes \boldsymbol{E}_{S} \otimes \boldsymbol{I}_{9}\right) \mathcal{P}_{F}^{(1)}, \\
& F T^{(2)}=\left(\mathbf{q}^{(2)}\right)^{T}\left(\boldsymbol{H}_{\eta} \otimes \boldsymbol{E}_{N} \otimes \boldsymbol{I}_{9}\right) \mathcal{F}_{\xi}^{(2)}+2\left(\mathbf{q}^{(2)}\right)^{T}\left(\boldsymbol{H}_{\xi} \otimes \boldsymbol{E}_{N} \otimes \boldsymbol{I}_{9}\right) \mathcal{P}_{F}^{(2)}
\end{aligned}
$$

The boundary terms are defined similarly, for instance the west boundary term is

$$
B T_{W}^{(l)}=-\left(\mathbf{q}^{(l)}\right)^{T}\left(\boldsymbol{E}_{W} \otimes \boldsymbol{H}_{\eta} \otimes \boldsymbol{I}_{9}\right) \mathcal{F}_{\xi}^{(l)}+2\left(\mathbf{q}^{(l)}\right)^{T}\left(\boldsymbol{E}_{W} \otimes \boldsymbol{H}_{\eta} \otimes \boldsymbol{I}_{9}\right) \mathcal{P}_{W}^{(l)} .
$$

The fault and boundary terms both arise in the continuous energy estimate (41) in the form of integrals along the fault and boundaries.

Recall that in the energy balance for the continuous problem, an integral term over the interior domain (the term in (41) multiplied by $(1-2 \gamma)$ ), canceled due to the chain rule. In contrast, this cancellation does not occur in the discrete problem 
since the difference operators to not satisfy the chain rule. This leads to the growth rate term

$$
\begin{aligned}
G R_{\alpha}^{(l)}=(1-2 \gamma) & {\left[\left(\mathbf{q}^{(l)}\right)^{T}\left(\boldsymbol{H}_{\xi} \otimes \boldsymbol{H}_{\eta} \otimes \boldsymbol{I}_{9}\right) \boldsymbol{D}_{\alpha} \mathcal{F}_{\alpha}^{(l)}\right.} \\
& \left.-\left(\boldsymbol{D}_{\alpha} \mathbf{q}^{(l)}\right)^{T}\left(\boldsymbol{H}_{\xi} \otimes \boldsymbol{H}_{\eta} \otimes \boldsymbol{I}_{9}\right) \mathcal{F}_{\alpha}^{(l)}\right] .
\end{aligned}
$$

This term does not appear in the continuous energy estimate (41) and, as we demonstrate later in the results section, can lead to unphysical energy growth and loss of accuracy of the numerical solution. The problem is most pronounced for highly distorted meshes. Fortunately, by setting $\gamma=1 / 2, G R_{\alpha}^{(l)}=0$. The growth term does not vanish for other values of $\gamma$, including $\gamma=0$, which puts the formulation into the widely used conservation form). A further discussion of the $\gamma=0$ case can be found in Nordström and Carpenter (2001) and Nordström (2006).

Remark: In the cancellation of the unphysical growth rate term, no assumption was made about the form of the operators $\boldsymbol{A}_{x}$ and $\boldsymbol{A}_{y}$, and thus a similar procedure will work for any constant coefficient hyperbolic system.

Considering the fault terms (60) and (61) we have that

$$
\begin{aligned}
& F T^{(1)}=-\left(\mathbf{v}_{j}^{(1)}\right)_{F}^{T} \boldsymbol{H}_{\xi} \boldsymbol{J}_{F}^{(1)} \frac{\partial \boldsymbol{\eta}^{(1)}}{\partial x_{i}}\left(\boldsymbol{\sigma}_{i j}^{(1)}\right)_{F}+2\left(\mathbf{q}_{F}^{(1)}\right)^{T}\left(\boldsymbol{H}_{\xi} \otimes \boldsymbol{I}_{9}\right) \tilde{\mathcal{P}}_{F}^{(1)}, \\
& F T^{(2)}=\left(\mathbf{v}_{j}^{(2)}\right)_{F}^{T} \boldsymbol{H}_{\xi} \boldsymbol{J}_{F}^{(2)} \frac{\partial \boldsymbol{\eta}^{(2)}}{\partial x_{i}}\left(\boldsymbol{\sigma}_{i j}^{(2)}\right)_{F}+2\left(\mathbf{q}_{F}^{(2)}\right)^{T}\left(\boldsymbol{H}_{\xi} \otimes \boldsymbol{I}_{9}\right) \tilde{\mathcal{P}}_{F}^{(2)},
\end{aligned}
$$

where the matrices are defined as

$$
\boldsymbol{J}_{F}^{(l)}=\operatorname{diag}\left[\begin{array}{lll}
J_{00}^{(l)} & \ldots & J_{N_{\xi} 0}^{(l)}
\end{array}\right]
$$

and only contain the values along the fault boundary. The same applies to $\partial \boldsymbol{\eta}^{(l)} / \partial x_{i}$. The vectors are defined as

$$
\left(\mathbf{v}_{j}^{(1)}\right)_{F}=\left(\boldsymbol{I}_{\xi} \otimes \mathbf{e}_{S}^{T}\right) \mathbf{v}_{j}^{(1)}, \quad\left(\mathbf{v}_{j}^{(2)}\right)_{F}=\left(\boldsymbol{I}_{\xi} \otimes \mathbf{e}_{N}^{T}\right) \mathbf{v}_{j}^{(2)},
$$

with analogous definitions for $\left(\boldsymbol{\sigma}_{i j}^{(l)}\right)_{F}, \mathbf{q}_{F}^{(l)}$, and $\tilde{\mathcal{P}}_{F}^{(l)}$. If we rewrite the variables in terms of the characteristic variables and pick the penalty terms such that

$$
\begin{aligned}
\left(\boldsymbol{\Sigma}_{F}^{(l)}\right)_{i 0}^{T} \mathbf{q}_{i 0}^{(l)} & =-\frac{1}{4} J_{i 0}^{(l)}\left|\nabla \eta_{i 0}^{(l)}\right|\left(\boldsymbol{Z}^{(l)}\right)^{-1} \mathbf{w}_{i 0}^{-(l)}, \\
\boldsymbol{Z}^{(l)} & =\operatorname{diag}\left[Z_{p}^{(l)}, Z_{s}^{(l)}, Z_{s}(l)\right]
\end{aligned}
$$

the energy dissipation rate at the fault is

$$
\begin{aligned}
F T^{(1)}+F T^{(2)}=\frac{1}{4} \sum_{l=1}^{2} & {\left[\left(\mathbf{w}_{F}^{+(l)}-\mathbf{w}_{F}^{-(l)}\right)^{T} \boldsymbol{B}_{F}^{(l)}\left(\mathbf{w}_{F}^{+(l)}+\mathbf{w}_{F}^{-(l)}\right)\right.} \\
& \left.-2\left(\mathbf{w}_{F}^{-(l)}\right)^{T} \boldsymbol{B}_{F}^{(l)}\left(\mathbf{w}_{F}^{-(l)}-\mathcal{W}_{F}^{-(l)}\right)\right]
\end{aligned}
$$


with matrices defined as

$$
\begin{aligned}
\boldsymbol{B}_{F}^{(l)} & =\boldsymbol{H}_{\xi} \boldsymbol{J}_{F}^{(l)}\left|\boldsymbol{\nabla} \eta^{(l)}\right|_{F} \otimes\left(\boldsymbol{Z}^{(l)}\right)^{-1}, \\
\left|\boldsymbol{\nabla} \eta^{(l)}\right|_{F} & =\operatorname{diag}\left[\left|\boldsymbol{\nabla} \eta^{(l)}\right|_{00}^{(l)} \ldots\left|\boldsymbol{\nabla} \eta^{(l)}\right|_{N_{\xi} 0}^{(l)}\right] .
\end{aligned}
$$

Since we exclusively consider the diagonal norm SBP operators, $\boldsymbol{B}_{F}^{(l)}$ is (diagonal) symmetric positive definite and (70) can be written as

$$
\begin{aligned}
& F T^{(1)}+F T^{(2)}=-\frac{1}{4} \sum_{l=1}^{2} {\left[\left(\mathbf{w}_{F}^{+(l)}-\mathcal{W}_{F}^{-(l)}\right)^{T} \boldsymbol{B}_{F}^{(l)}\left(\mathbf{w}_{F}^{+(l)}+\mathcal{W}_{F}^{-(l)}\right)\right.} \\
&\left.+\left(\mathbf{w}_{F}^{-(l)}-\mathcal{W}_{F}^{-(l)}\right)^{T} \boldsymbol{B}_{F}^{(l)}\left(\mathbf{w}_{F}^{-(l)}-\mathcal{W}_{F}^{-(l)}\right)\right] .
\end{aligned}
$$

Additionally, just as in the continuous problem we have that $\boldsymbol{J}_{F}^{(1)}\left|\nabla \eta^{(1)}\right|_{F}=$ $\boldsymbol{J}_{F}^{(2)}\left|\boldsymbol{\nabla} \eta^{(2)}\right|_{F}$. In order to show that (73) is negative semidefinite we define the stresses

$$
\begin{array}{ll}
\hat{\boldsymbol{\sigma}}^{(1)}=\frac{1}{2}\left(\mathcal{W}_{P}^{-(1)}+\mathbf{w}_{P}^{+(1)}\right)_{F}, & \hat{\boldsymbol{\sigma}}^{(2)}=-\frac{1}{2}\left(\mathcal{W}_{P}^{-(2)}+\mathbf{w}_{P}^{+(2)}\right)_{F}, \\
\hat{\boldsymbol{\tau}}_{m}^{(1)}=-\frac{1}{2}\left(\mathcal{W}_{S V}^{-(1)}+\mathbf{w}_{S V}^{+(1)}\right)_{F}, & \hat{\boldsymbol{\tau}}_{m}^{(2)}=\frac{1}{2}\left(\mathcal{W}_{S V}^{-(2)}+\mathbf{w}_{S V}^{+(2)}\right)_{F}, \\
\hat{\boldsymbol{\tau}}_{z}^{(1)}=-\frac{1}{2}\left(\mathcal{W}_{S H}^{-(1)}+\mathbf{w}_{S H}^{+(1)}\right)_{F}, & \hat{\boldsymbol{\tau}}_{z}^{(2)}=\frac{1}{2}\left(\mathcal{W}_{S H}^{-(2)}+\mathbf{w}_{S H}^{+(2)}\right)_{F},
\end{array}
$$

and velocities

$$
\begin{aligned}
\hat{\boldsymbol{\beta}} & =\frac{1}{2 Z_{p}^{(1)}}\left(\mathcal{W}_{P}^{-(1)}-\mathbf{w}_{P}^{+(1)}\right)_{F}-\frac{1}{2 Z_{p}^{(2)}}\left(\mathcal{W}_{P}^{-(2)}-\mathbf{w}_{P}^{+(2)}\right)_{F}, \\
\hat{\mathbf{V}}_{m} & =\frac{1}{2 Z_{s}^{(1)}}\left(\mathcal{W}_{S V}^{-(1)}-\mathbf{w}_{S V}^{+(1)}\right)_{F}-\frac{1}{2 Z_{s}^{(2)}}\left(\mathcal{W}_{S V}^{-(2)}-\mathbf{w}_{S V}^{+(2)}\right)_{F}, \\
\hat{\mathbf{V}}_{z} & =\frac{1}{2 Z_{s}^{(1)}}\left(\mathcal{W}_{S H}^{-(1)}-\mathbf{w}_{S H}^{+(1)}\right)_{F}-\frac{1}{2 Z_{s}^{(2)}}\left(\mathcal{W}_{S H}^{-(2)}-\mathbf{w}_{S H}^{+(2)}\right)_{F} .
\end{aligned}
$$

Since $\mathcal{W}^{-(l)}$ is derived from fault conditions (33), (37), and (38), it follows that these stresses and velocities satisfy the same conditions:

$$
\begin{aligned}
\hat{\boldsymbol{\sigma}}^{(1)} & =-\hat{\boldsymbol{\sigma}}^{(2)}, & \hat{\boldsymbol{\tau}}_{m}^{(1)}=-\hat{\boldsymbol{\tau}}_{m}^{(2)}, & \hat{\boldsymbol{\tau}}_{z}^{(1)}=-\hat{\boldsymbol{\tau}}_{z}^{(2)}, \\
\hat{\tau}_{i 0} & =f\left(\hat{V}_{i 0}, \psi_{i}\right) \hat{\sigma}_{i 0}, & \hat{\beta}_{i 0} & =0
\end{aligned}
$$

with

$$
\hat{\tau}_{i 0}=\sqrt{\left(\hat{\tau}_{m}^{(l)}\right)_{i 0}^{2}+\left(\hat{\tau}_{z}^{(l)}\right)_{i 0}^{2}}, \quad \hat{V}_{i 0}=\sqrt{\left(\hat{V}_{m}\right)_{i 0}^{2}+\left(\hat{V}_{z}\right)_{i 0}^{2}}
$$


With this notation the fault energy dissipation rate (70) becomes

$$
\begin{aligned}
F T^{(1)}+F T^{(2)}= & (\hat{\boldsymbol{\beta}})^{T} \tilde{\boldsymbol{B}}_{F}^{(2)} \hat{\boldsymbol{\sigma}}-\left(\hat{\mathbf{V}}_{m}\right)^{T} \tilde{\boldsymbol{B}}_{F}^{(2)} \hat{\boldsymbol{\tau}}_{m}-\left(\hat{\mathbf{V}}_{z}\right)^{T} \tilde{\boldsymbol{B}}_{F}^{(2)} \hat{\boldsymbol{\tau}}_{z} \\
& -\frac{1}{4} \sum_{l=1}^{2}\left(\mathbf{w}_{F}^{-(l)}-\boldsymbol{W}_{F}^{-(l)}\right)^{T} \boldsymbol{B}_{F}^{(l)}\left(\mathbf{w}_{F}^{-(l)}-\boldsymbol{W}_{F}^{-(l)}\right) \\
= & -(\hat{\mathbf{V}})^{T} \tilde{\boldsymbol{B}}_{F}^{(2)} \operatorname{diag}[\mathbf{f}(\hat{\mathbf{V}}, \boldsymbol{\psi})] \hat{\boldsymbol{\sigma}} \\
& -\frac{1}{4} \sum_{l=1}^{2}\left(\mathbf{w}_{F}^{-(l)}-\boldsymbol{W}_{F}^{-(l)}\right)^{T} \boldsymbol{B}_{F}^{(l)}\left(\mathbf{w}_{F}^{-(l)}-\mathcal{W}_{F}^{-(l)}\right),
\end{aligned}
$$

where

$$
\tilde{\boldsymbol{B}}_{F}^{(l)}=\boldsymbol{H}_{\xi} \boldsymbol{J}_{F}^{(l)}\left|\nabla \eta^{(l)}\right|_{F},
$$

$\tilde{\boldsymbol{B}}_{F}^{(1)}=\tilde{\boldsymbol{B}}_{F}^{(2)}$, and $\mathbf{f}(\hat{\mathbf{V}}, \boldsymbol{\psi})$ denotes the vector of friction coefficients evaluated at components of $\hat{\mathbf{V}}$. Since $\tilde{\boldsymbol{B}}_{F}^{(l)}$ is diagonal and positive definite

$$
(\hat{\mathbf{V}})^{T} \tilde{\boldsymbol{B}}_{F}^{(2)} \operatorname{diag}[\mathbf{f}(\hat{\mathbf{V}}, \boldsymbol{\psi})] \hat{\boldsymbol{\sigma}} \geq 0
$$

overall (83) is negative semidefinite and there is no energy growth due to the fault terms. The boundary term is of the same form as in the continuous problem (see (43)) with the addition of a small numerical dissipation term that vanishes if the grid values exactly satisfy the friction law, as occurs with mesh refinement.

A similar calculation can be performed for the boundary terms. For example, considering the west boundary term (62) and picking the penalty vectors

$$
\left(\boldsymbol{\Sigma}_{W}^{(l)}\right)_{0 j}^{T} \mathbf{q}_{0 j}^{(l)}=-\frac{1}{4} J_{0 j}^{(l)}\left|\boldsymbol{\nabla} \xi_{0 j}^{(l)}\right|\left(\boldsymbol{Z}^{(l)}\right)^{-1} \mathbf{w}_{0 j}^{-(l)},
$$

we have the energy dissipation rate

$$
\begin{aligned}
B T_{W}^{(l)}= & \frac{1}{4}\left(\mathbf{w}_{W}^{-(l)}-\mathbf{w}_{W}^{+(l)}\right)^{T} \boldsymbol{B}_{W}^{(l)}\left(\mathbf{w}_{W}^{-(l)}+\mathbf{w}_{W}^{+(l)}\right)^{T} \\
& +\frac{1}{2}\left(\mathbf{w}_{W}^{-(l)}\right)^{T} \boldsymbol{B}_{W}^{(l)}\left(\mathbf{w}_{W}^{-(l)}-\boldsymbol{R}_{W}^{(l)} \mathbf{w}_{W}^{+(l)}\right) \\
= & -\frac{1}{4}\left(\mathbf{w}_{W}^{+(l)}\right)^{T}\left(\boldsymbol{B}_{W}^{(l)}-\boldsymbol{R}_{W}^{(l)} \boldsymbol{B}_{W}^{(l)} \boldsymbol{R}_{W}^{(l)}\right)\left(\mathbf{w}_{W}^{+(l)}\right) \\
& -\frac{1}{4}\left(\mathbf{w}_{W}^{-(l)}-\boldsymbol{R}_{W}^{(l)} \mathbf{w}_{W}^{+(l)}\right)^{T} \boldsymbol{B}_{W}^{(l)}\left(\mathbf{w}_{W}^{-(l)}-\boldsymbol{R}_{W}^{(l)} \mathbf{w}_{W}^{+(l)}\right),
\end{aligned}
$$

where

$$
\begin{aligned}
\boldsymbol{B}_{W}^{(l)} & =\boldsymbol{H}_{\eta} \boldsymbol{J}_{W}^{(l)}|\boldsymbol{\nabla} \boldsymbol{\xi}|_{W}^{(l)} \otimes\left(\boldsymbol{Z}^{(l)}\right)^{-1}, \\
|\boldsymbol{\nabla} \boldsymbol{\xi}|_{W}^{(l)} & =\operatorname{diag}\left[\left|\nabla \xi_{00}^{(1)}\right| \ldots\left|\nabla \xi_{0 N_{\eta}}^{(1)}\right|\right], \\
|\nabla \boldsymbol{\xi}|_{W}^{(2)} & =\operatorname{diag}\left[\left|\nabla \xi_{0,-N_{\eta}}^{(2)}\right| \ldots\left|\nabla \xi_{00}^{(2)}\right|\right] .
\end{aligned}
$$


Equation (87) is negative definite if $\mathbf{B}_{W}^{(l)}$ and $\mathbf{B}_{W}^{(l)}-\mathbf{R}_{W} \mathbf{B}_{W}^{(l)} \mathbf{R}_{W}$ are positive semidefinite which follows from the diagonal norm SBP operators and the boundedness of the reflection coefficients in \pm 1 . As in the case of the fault, the energy dissipation rate at the exterior boundary is the same as in the continuous problem (see (42)) with the addition of a small numerical dissipation term that vanishes with mesh refinement.

We can now state the following proposition:

Proposition 2 The discrete scheme (44) is stable.

\section{Results}

We test the method in two ways. The first uses the method of manufactured solutions (MMS) (Roache, 1998). In MMS, boundary data (forcing functions) and source terms are added to the problem in such a way that the exact solution is known a priori. This permits a rigorous convergence test that establishes the order of accuracy of the method. In the second test we apply the method to an extremely challenging application problem - a subduction zone megathrust earthquake - involving a highly irregular domain, complex free surface topography, multiple blocks with different material properties, and a nonplanar fault.

In all the results that follow, the mesh is generated using the transfinite interpolation method (Knupp and Steinberg, 1993) after defining location of the points (and outward pointing normals) on the boundaries and faults. The metric derivatives (e.g., $\partial x / \partial \xi$ ) are computed using the same SBP difference operators used in the semi-discrete approximation. It is easy to verify that the use of the discrete metric derivatives has no effect on the stability of the method. Additionally, since the same SBP operators are used, the accuracy of the method remains the same.

As formulated, the method can encompass all two-dimensional problems, including both plane strain (mode II ruptures), antiplane shear (mode III ruptures), and even mixed mode shear ruptures. However, both tests are restricted to plane strain deformation in the $x$ - and $y$-directions only, for which only $\mathrm{P}$ and $\mathrm{SV}$ waves are excited.

\subsection{Rate-and-State Friction and Nondimensionalization}

Before proceeding to the computational results, we first present the specific rateand-state friction law used in this work and cast the problems in nondimensional form.

We use the regularized slip law form of rate-and-state friction (Rice, 1983; Lapusta et al., 2000):

$$
f(V, \psi)=a \operatorname{arcsinh}\left(\frac{V}{2 V_{0}} \exp \left(\frac{\psi}{a}\right)\right), \frac{d \psi}{d t}=-\frac{V}{L}\left[f(V, \psi)-f_{s s}(V)\right],
$$

where $L$ is the state evolution distance, $V_{0}$ is an arbitrary reference velocity, and $a$ is the direct effect parameter. Experiments and theory demonstrate that $a>0$ (Rice et al., 2001), corresponding to an instantaneous strengthening of the fault following an abrupt increase in $V$, as illustrated in Figure 1. Following the direct 
effect, $f(V, \psi)$ evolves toward $f_{s s}(V)$ over a characteristic slip distance $L$. For the steady state friction coefficient, we use the standard logarithmic form

$$
f_{s s}(V)=f_{0}-(b-a) \ln \left(\frac{V}{V_{0}}\right)
$$

where $f_{0}$ is the steady state friction coefficient at $V=V_{0}$ and the sign of $b-a$ determines whether the fault is velocity-strengthening $(b-a<0)$ or velocityweakening $(b-a>0)$ in steady state. The latter is necessary for unstable slip and self-sustaining rupture propagation.

We next nondimensionalize the equations using the assumption that at least some portion of the fault is velocity-weakening in steady state (so $b-a>0$ ). The state evolution distance, $L$, provides a characteristic slip distance. A linear stability analysis of steady sliding along a planar frictional interface with $b-a>0$ between identical elastic half-spaces (Rice and Ruina, 1983; Rice et al., 2001) reveals that pertubations having along-fault wavelengths exceeding a critical wavelength are unstable; shorter wavelength perturbations are stable. (And if $b-a<0$ then the interface is stable to all perturbations as long as $a \geq 0$.) For quasi-static elasticity, the critical wavelength is approximately $h^{*}=G^{(1)} L /(b-a) \sigma_{n}^{0}$, and we take this as the characteristic distance scale. The associated time scale is $T=h^{*} / c_{s}^{(1)}$. The grid spacing (along the fault) must be smaller than $h^{*}$ so that the unresolved (Nyquist) modes in the solutions cannot trigger this physical instability.

For propagating ruptures, inertial terms become important in the momentum balance and $h^{*}$ no longer provides an accurate characterization of spatial variations in the solution. In particular, fields vary most rapidly in the region immediately behind the propagating rupture front, where the strength drop occurs. The strength drop occurs over a distance, $R$, that is proportional to $h^{*}$, but about three orders of magnitude smaller than $h^{*}$ for typical parameters. For more discussion of $R$ and $h^{*}$, see Rice et al. (2001) and Dunham et al. (in press).

We therefore nondimensionalize space and time as

$$
\bar{x}=\frac{x}{h^{*}}, \bar{y}=\frac{y}{h^{*}}, \bar{t}=\frac{t}{T}
$$

The nondimensional grid spacing must satisfy $\bar{h} \ll 1$ (in the vicinity of the fault). Stresses and particle velocities are related via the shear impedance, $Z_{s}^{(1)}$, leading to the nondimensionalization

$$
\bar{\sigma}_{i j}=\frac{\sigma_{i j}}{(b-a) \sigma_{n}^{0}}, \quad \bar{v}_{i}=\frac{Z_{s}^{(1)} v_{i}}{(b-a) \sigma_{n}^{0}} \quad \text { and } \quad \bar{V}=\frac{Z_{s}^{(1)} V}{2(b-a) \sigma_{n}^{0}} .
$$

For the friction law, we select the reference slip velocity as $V_{0}=2(b-a) \sigma_{n}^{0} / Z_{s}^{(1)}$, which leads to the dimensionless form

$$
\begin{aligned}
f & =a \operatorname{arcsinh}\left(\frac{\bar{V}}{2} \exp \left(\frac{\psi}{a}\right)\right), \\
\frac{d \psi}{d \bar{t}} & =-\bar{V}\left[f(\bar{V}, \psi)-f_{s s}(\bar{V})\right], f_{s s}(\bar{V})=f_{0}-(b-a) \ln (\bar{V}) .
\end{aligned}
$$

Thus in the friction law we have three nondimensional parameters: $a, b-a$, and $f_{0}$. The equations also require an initial value for $\psi$. 
In the numerical integration of the state variable $\psi$, the "hat" variables along the fault interface, as defined in (74)-(79), are used. This is done so that state and the interface relations are integrated in a consistent manner. Additionally, this avoids any complications with the fact that the traction components of stress, as defined by the grid values, are not necessarily continuous across the interface when the SAT method is used.

\subsection{Method of Manufactured Solutions}

We next construct an MMS solution that satisfies continuity of traction components of stress across the fault $\left(\bar{T}_{i}^{(1)}=-\bar{T}_{i}^{(2)}\right)$, the no-opening condition $(\bar{\beta}=0)$, and parallel slip velocity and shear stress. (Of course, this is not strictly necessary, but facilitates verification of our implementation). Since we only consider in-plane deformation, $\bar{\tau}_{z}=\bar{V}_{z}=0$, and the parallel requirement reduces to $\operatorname{sign}\left(\bar{V}_{m}\right)=\operatorname{sign}\left(\bar{\tau}_{m}\right)$.

The fault is given by the curve $\bar{y}=\kappa(\bar{x})$ having unit normal and perpendicular vectors

$$
\begin{gathered}
\mathbf{n}^{(1)}=-\mathbf{n}^{(2)}=\frac{1}{\sqrt{1+\kappa^{\prime}(\bar{x})^{2}}}\left[\begin{array}{c}
\kappa^{\prime}(\bar{x}) \\
-1
\end{array}\right], \\
\mathbf{m}^{(1)}=-\mathbf{m}^{(2)}=\frac{-1}{\sqrt{1+\kappa^{\prime}(\bar{x})^{2}}}\left[\begin{array}{c}
1 \\
\kappa^{\prime}(\bar{x})
\end{array}\right] .
\end{gathered}
$$

To define the solution we introduce the following auxiliary functions:

$$
\varsigma_{n n}(\bar{x}, \bar{y}, \bar{t}), \varsigma_{m m}^{(l)}(\bar{x}, \bar{y}, \bar{t}), \varsigma_{n m}(\bar{x}, \bar{y}, \bar{t}), \varphi_{n}(\bar{x}, \bar{y}, \bar{t}), \varphi_{m}^{(l)}(\bar{x}, \bar{y}, \bar{t}),
$$

which are all continuous function of space and time, except for discontinuities in $\varsigma_{m m}^{(l)}$ and $\varphi_{m}^{(l)}$ across the fault. The solution in the entire domain is

$$
\begin{aligned}
& \bar{\sigma}_{x x}^{(l)}=-\varsigma_{n n} m_{y}^{2}+\varsigma_{m m}^{(l)} n_{y}^{2}-2 \varsigma_{n m} m_{y} n_{y}, \\
& \bar{\sigma}_{y y}^{(l)}=-\varsigma_{n n} m_{x}^{2}+\varsigma_{m m}^{(l)} n_{x}^{2}-2 \varsigma_{n m} m_{x} n_{x}, \\
& \bar{\sigma}_{x y}^{(l)}=\varsigma_{n n} m_{x} m_{y}-\varsigma_{m m}^{(l)} n_{x} n_{y}+\varsigma_{n m}\left(n_{y} m_{x}+n_{x} m_{y}\right), \\
& \bar{v}_{x}^{(l)}=\varphi_{m}^{(l)} n_{y}-\varphi_{n} m_{y}, \quad \bar{v}_{y}^{(l)}=-\varphi_{m}^{(l)} n_{x}+\varphi_{n} m_{x}, \\
& m_{x}=-n_{y}=\frac{1}{\sqrt{1+\kappa^{\prime}(\bar{x})^{2}}}, \quad m_{y}=n_{x}=\frac{\kappa^{\prime}(\bar{x})}{\sqrt{1+\kappa^{\prime}(\bar{x})^{2}}} .
\end{aligned}
$$

It is easy to verify, that this solution yields fault values: $\bar{\beta}=0, \bar{\tau}=\varsigma_{n m}, \bar{\sigma}_{n}=-\varsigma_{n n}$, and $\bar{V}=\varphi_{m}^{(1)}-\varphi_{m}^{(2)}$.

To force the solution at the fault we do not specify the state variable directly (except through the initial conditions), but instead add a source term to the state evolution equation (96). This has the added benefit of verifying the numerical method and implementation not only with a nonlinear interface conditions but also with state evolution. The modified state evolution equation is

$$
\begin{aligned}
\frac{d \psi}{d \bar{t}} & =-\bar{V}\left[f(\bar{V}, \psi)-f_{s s}(\bar{V})\right]+s, \\
s & =\bar{V}^{*}\left[f\left(\bar{V}^{*}, \psi^{*}\right)-f_{s s}\left(\bar{V}^{*}\right)\right]+\frac{d \psi^{*}}{d t}, \quad \psi^{*}=\ln \left(\frac{2}{\bar{V}^{*}} \sinh \left(\frac{\bar{\tau}^{*}}{a \bar{\sigma}_{n}^{*}}\right)\right),
\end{aligned}
$$




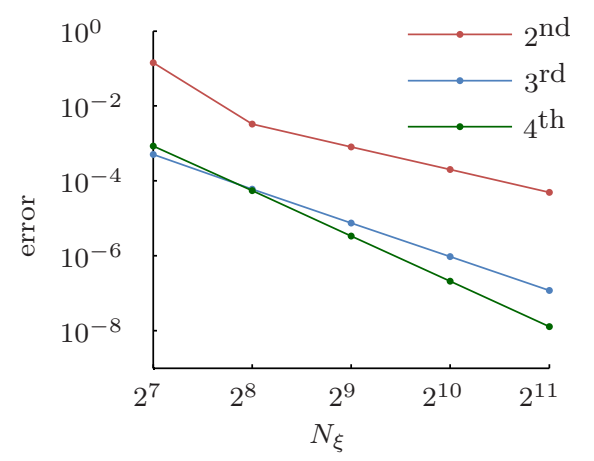

\begin{tabular}{rcccccc}
\hline & \multicolumn{2}{c}{$2^{\text {nd }}$} & \multicolumn{2}{c}{$3^{\text {rd }}$} & \multicolumn{2}{c}{$4^{\text {th }}$} \\
$N_{\xi}$ & error & rate & error & rate & error & rate \\
\hline $2^{7}$ & $1.42 \times 10^{-1}$ & $\ldots$ & $5.05 \times 10^{-4}$ & $\cdots$ & $8.49 \times 10^{-4}$ & $\ldots$ \\
$2^{8}$ & $3.28 \times 10^{-3}$ & 5.43 & $6.04 \times 10^{-5}$ & 3.06 & $5.48 \times 10^{-5}$ & 3.95 \\
$2^{9}$ & $8.02 \times 10^{-4}$ & 2.03 & $7.52 \times 10^{-6}$ & 3.00 & $3.38 \times 10^{-6}$ & 4.02 \\
$2^{10}$ & $1.99 \times 10^{-4}$ & 2.01 & $9.44 \times 10^{-7}$ & 2.99 & $2.08 \times 10^{-7}$ & 4.02 \\
$2^{11}$ & $4.97 \times 10^{-5}$ & 2.00 & $1.18 \times 10^{-7}$ & 3.00 & $1.29 \times 10^{-8}$ & 4.01 \\
\hline
\end{tabular}

Fig. 3: Error and convergence rate estimates for the method of manufactured solutions.

where the superscript $*$ indicates fields that are known functions of space and time evaluated using the exact solution (100)-(103). For the outer boundary conditions we specify the incoming characteristic variables, namely $\mathbf{w}^{-(l)}=\mathbf{g}$ on $\partial \Omega$ where $\mathrm{g}$ is easily defined from (100)-(103) using the outward pointing normal.

For our test we define the auxiliary functions to be

$$
\begin{aligned}
\varsigma_{n n} & =-g(\bar{x}, \bar{y}, \bar{t})+\varsigma_{n n}^{0}, & \varsigma_{m m}^{(l)} & =(-1)^{l} g(\bar{x}, \bar{y}, \bar{t}), \\
\varsigma_{n m} & =g(\bar{x}, \bar{y}, \bar{t})+\varsigma_{n m}^{0}, & \varphi_{n} & =g(\bar{x}, \bar{y}, \bar{t}), \\
\varphi_{m}^{(l)} & =(-1)^{l}\left[g(\bar{x}, \bar{y}, \bar{t})+\varphi_{m}^{0}\right], & g(\bar{x}, \bar{y}, \bar{t}) & =\cos (\bar{k} \bar{x}) \cos (\bar{k} \bar{y}) \cos (\bar{\omega} \bar{t}),
\end{aligned}
$$

with $\bar{k}=2 \pi, \bar{\omega}=100 \pi / 69, \varsigma_{n n}^{0}=-250, \varsigma_{n m}^{0}=150$, and $\varphi_{m}^{0}=4000 / 63 \approx 63.5$. The domain for this test is $-1 \leq \bar{x}, \bar{y} \leq 1$ and the fault is $\bar{y}=\sin (\pi \bar{x})$. The simulation is run for grid resolutions $N_{\xi}=2 N_{\eta}=2^{i}$ where $i=7,8,9,10,11$ until $\bar{t}_{f}=13.8$ which corresponds to $\bar{t}_{f} \bar{\omega} / 2 \pi=10$ oscillations of the solution. The material is identical on both sides of the fault with a Poisson ratio of $\nu=0.21875$. Time integration is performed with a $4^{\text {th }}$ order, low memory Runge-Kutta method of Carpenter and Kennedy (1994) (their 5[4] method with solution 3) using a time step size $\Delta \bar{t}=0.3 \bar{h}_{\text {min }}$, where

$$
\bar{h}_{\min }=\min _{\xi, \eta}\left[\min \left(\frac{J}{N_{\xi} \sqrt{x_{\eta}^{2}+y_{\eta}^{2}}}, \frac{J}{N_{\eta} \sqrt{x_{\xi}^{2}+y_{\xi}^{2}}}\right)\right] .
$$


The error in the solution is defined as

$$
\operatorname{error}\left(N_{\xi}\right)=\left\|\mathbf{q}-\mathbf{q}^{*}\right\|_{h},
$$

where the norm $\|\cdot\|_{h}$ is the energy norm defined in (57) and $\mathbf{q}^{*}$ is the exact solution evaluated at the same spatial locations as the discrete solution. We estimate the convergence rate between successive solutions as

$$
\mathrm{p}\left(N_{\xi}\right)=\log _{2}\left(\frac{\operatorname{error}\left(N_{\xi} / 2\right)}{\operatorname{error}\left(N_{\xi}\right)}\right) .
$$

The results for the $2^{\text {nd }}, 3^{\text {rd }}$, and $4^{\text {th }}$ order diagonal SBP operators (Kreiss and Scherer, 1974, 1977; Strand, 1994) are given in Figure 3, which shows the error as a function of $N_{\xi}$ and the estimated convergence rates. The convergence rate approaches the theoretical limit under sufficient refinement.

\subsection{Subduction Zone Megathrust Earthquake}

We now consider a more complex application problem to demonstrate the full potential of the method. The problem is motivated by the recent magnitude 9.0 Tohoku-Oki, Japan, megathrust earthquake and the resulting tsunami. The specific geometry we consider is shown in Figure 6, and is loosely based on the subduction zone structure in the vicinity of the Japan trench (Tsuru et al., 2000; Takahashi et al., 2004). The Pacific Plate is being subducted to the west beneath the North American / Okhotsk Plate, with relative motion across the plate interface (the fault) occurring during megathrust earthquakes. The Japanese island of Honshu lies at the left edge of the domain, and the upper boundary of the entire computational domain is the seaoor (with the ocean deepening oshore until it reaches a maximum depth of about $7 \mathrm{~km}$ at the trench). Slip along the plate interface causes vertical deformation of the seaoor, causing uplift or subsidence of the overlying water layer. Gravity waves (tsunamis) occur as the sea surface returns to an equilibrium level.

The east (Pacific Plate) side is idealized with a two-layer model (oceanic crust and mantle). As the Pacific Plate dives beneath the North American / Okhotsk Plate, it crosses several material layers (idealized here as upper and lower crust and the mantle wedge). We do not include an ocean layer in this model, due to the large impedance contrast between water and rock, and instead approximate the seaoor as a traction-free surface. We do note, however, that the method as designed can easily be used for acoustic wave propagation in uids simply by setting the shear modulus to zero. The small angle between the seaoor and the fault (about $5^{\circ}$ ) creates an extremely challenging geometry.

For this problem, the method must be extended to handle multi-block interfaces. Instead of enforcing a friction law across the non-fault interfaces, continuity conditions are enforced using the characteristic variables that propagate across the interfaces. The penalty terms are of an identical form as those used for the fault interfaces. Figure 4 shows the multi-block structure used for this example, with solid lines representing interfaces across which there is a material contrast and dotted lines represent purely computational interfaces. In this model there are 20 blocks and 31 interfaces, six of which are frictional interfaces. 


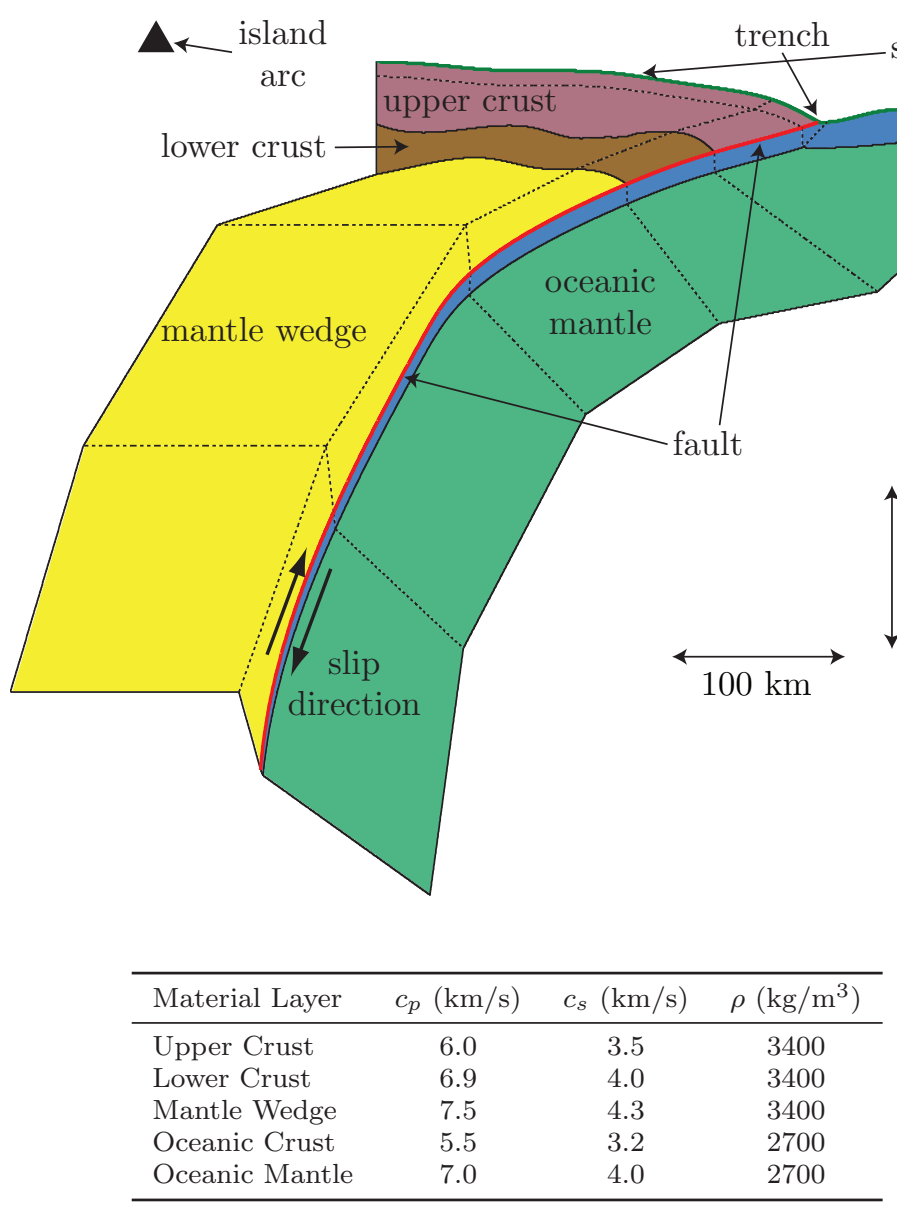

Fig. 4: Subduction zone geometry for megathrust earthquake problem, shown with a factor of 5.25 vertical exaggeration. Different materials are signified with different colors. Solid lines indicate boundaries between the different materials and dotted lines indicate the computational (multi-block grid) boundaries. The fault is highlighted with a thicker solid red line and the seafloor (traction-free surface) is represented with a solid green line. All other boundaries are absorbing boundaries. The accompanying table lists material properties.

Initial conditions are required on stress, velocity, and state variable. We take the initial velocity to be everywhere zero. We write the stress as the superposition of a prestress, $\sigma_{i j}^{0}$, and stress perturbations arising from slip on the fault, $\Delta \sigma_{i j}$. Linearity of the elastic wave equation permits us to solve only for stress perturbations in the interior, but the nonlinear friction law must be enforced using the absolute stress values. Hence we write the shear and normal stresses used in the friction law (38) as $\tau=\tau_{0}+\Delta \tau$ and $\sigma_{n}=\sigma_{0}+\Delta \sigma_{n}$, respectively, where the prestress values $\tau_{0}$ and $\sigma_{0}$ are known functions and the stress changes are calculated 


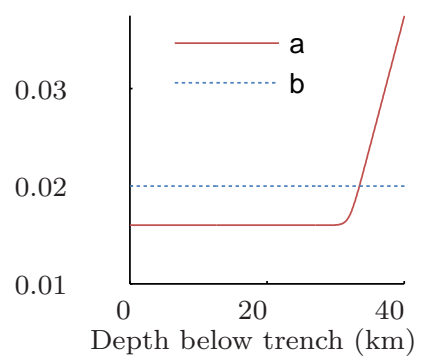

\begin{tabular}{llr}
\hline parameter & & value \\
\hline steady state friction at $V_{0}$ & $f_{0}$ & 0.3 \\
reference velocity & $V_{0}$ & $10^{-6} \mathrm{~m} / \mathrm{s}$ \\
initial state variable & $\psi_{0}$ & 0.5 \\
state evolution distance & $L$ & $0.8 \mathrm{~m}$ \\
\hline
\end{tabular}

Fig. 5: Frictional parameters for the subduction megathrust problem.

from wave propagation in the interior. This procedure is similar to adding forcing functions on the interface as done in the MMS test problem.

In general the prestress would be quite heterogeneous along the fault, depending on the overburden of the overlying rock, remote stressing, and previous earthquakes, but for simplicity we set the background normal stress to $\sigma_{0}=100 \mathrm{MPa}$ and take the initial shear stress to be $\tau_{0}=0.3 \sigma_{0}$ (except in the nucleation zone, as described below). Similarly, the friction parameters are expected to vary with temperature and rock type (and thus with depth), and in this model we use the simple profile given in Figure 5. Of course, more realistic parameter profiles can be constructed (e.g., Liu and Rice, 2005), but this is unnecessary for demonstrating the capabilities of the developed numerical method. With these values of $a$ and $b-a$, the rupture will propagate up the fault toward the free surface, but since $b-a$ becomes negative at depth, the downward rupture will eventually stop as the fault becomes velocity-strengthening.

In order to nucleate, or start, the rupture, the initial shear stress is increased over a small patch of the fault:

$$
\tau_{0}=0.3 \sigma_{0}+70 \mathrm{MPa} \exp \left[-\frac{\left(x-x_{0}\right)^{2}+\left(y-y_{0}\right)^{2}}{2(3 \mathrm{~km})^{2}}\right],
$$

where $y_{0}=30 \mathrm{~km}$ and $x_{0}=-245 \mathrm{~km}$. The stress perturbation immediately initiates rapid sliding via the direct effect (shear stress and slip velocity increase together), since state evolution is negligible over short time scales. Because the amplitude and width of the Gaussian perturbation are sufficiently large, the perturbation grows and leads to unstable slip and dynamic rupture.

To assess solution accuracy, we conduct the simulation at two levels of resolution. The low resolution run has $\sim 5 \times 10^{6}$ grid points (2123 in the $\xi$-direction and 2316 in the $\eta$-direction) with a minimum grid spacing along the fault, interfaces, and exterior boundaries of $100 \mathrm{~m}$, and interior minimum and maximum grid spacings $h_{\min }=0.19 \mathrm{~m}$ and $h_{\max }=200 \mathrm{~m}$, respectively, where $h_{\min }$ is defined in (110) and $h_{\max }$ is defined similarly. The time step is $\Delta t=1.5625 \times 10^{-4} \mathrm{~s}$, corresponding to an S-wave CFL of 0.35 . The $200 \mathrm{~s}$ simulation requires $1.28 \times 10^{6}$ time steps. The high resolution run has twice the grid resolution $\left(\sim 10^{7}\right.$ grid points and $2.56 \times 10^{6}$ time steps).

Figure 6(a) shows the wavefield at $15 \mathrm{~s}$. The relative motion of the North American / Okhotsk Plate and the Pacific Plate is consistent with the sense of 
(a) horizontal particle velocity $(\mathrm{m} / \mathrm{s})$ at $\mathrm{t}=15 \mathrm{~s}$

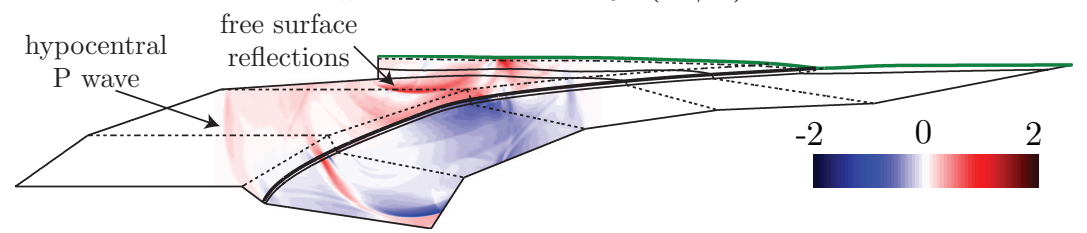

vertical particle velocity $(\mathrm{m} / \mathrm{s})$ at $\mathrm{t}=15 \mathrm{~s}$

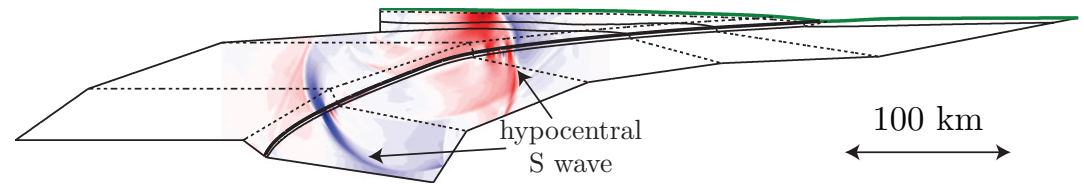

(b)

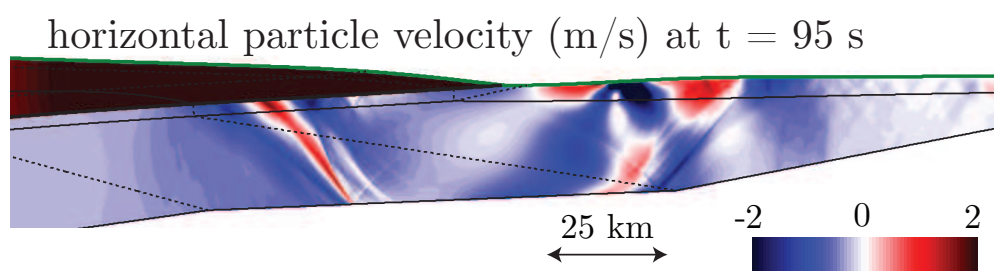

vertical particle velocity $(\mathrm{m} / \mathrm{s})$ at $\mathrm{t}=95 \mathrm{~s}$

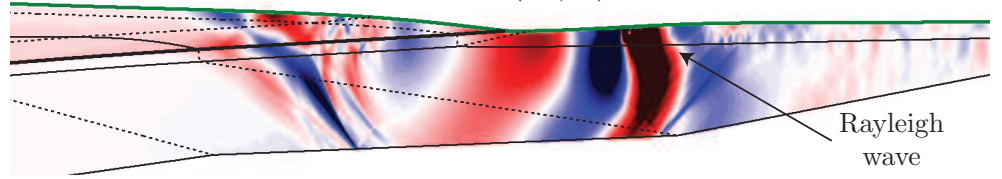

Fig. 6: (a) Wavefield at $t=15 \mathrm{~s}$ for the subduction zone megathrust earthquake. The actively slipping part of the fault lies slightly behind the hypocentral S-wave front. (b) Wavefield at $t=95 \mathrm{~s}$ shortly after the rupture has reached the trench. Figures are to scale with no vertical exaggeration. Note different scale bars in (a) and (b).

slip indicated in Figure 4. For the Japan trench, the island arc would be on the North American / Okhotsk Plate side, approximately $250 \mathrm{~km}$ from the trench axis (the intersection of the fault with the seafloor). Figure 6(b) shows the wavefield at $95 \mathrm{~s}$, shortly after the rupture has reached the trench. The material on the North American / Okhotsk Plate side is moving rapidly to the right, due to an extreme reduction in normal stress (and thus fault strength) from wave reflections off the seafloor. The wavefield is quite rich in structure, and includes dispersed Rayleigh waves propagating along the seafloor in the oceanic layers. These details are lost in more simplified models that simplify the physics and geometric complexity.

An important seismological quantity is the cumulative slip on the fault, which partially determines the magnitude of the earthquake. Slip is often estimated in inversion studies using geodetic and seismic data. We show the cumulative slip up to $95 \mathrm{~s}$, plotted every $5 \mathrm{~s}$, in Figure 7 for the two resolution levels. Results from 


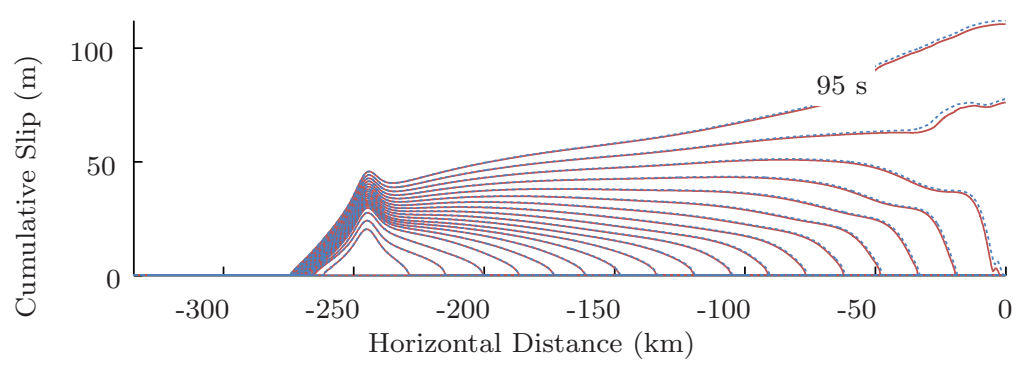

Fig. 7: Cumulative slip up to $95 \mathrm{~s}$ as a function of distance along the fault, plotted at $5 \mathrm{~s}$ intervals. The solid red line is the base resolution simulation and the dotted blue line is a simulation at twice the resolution. The earthquake is nucleated at approximately $-240 \mathrm{~km}$ along the fault and the trench/free surface is at $0 \mathrm{~km}$.

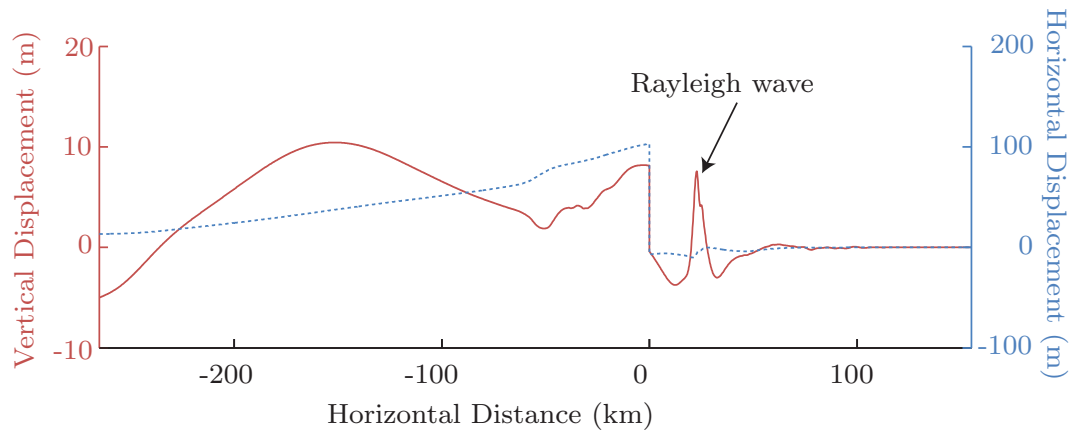

Fig. 8: Vertical (solid red line) and horizontal (dotted blue line) seafloor displacement at $t=95 \mathrm{~s}$ as a function of horizontal distance (see Figure 6). The fault reaches the seafloor at $0 \mathrm{~km}$.

the two simulations match to within $2.5 \%$, on average, with the largest errors (up to $200 \%$ ) around the rupture tip. These large errors around the rupture tip are not surprising as this is where the fault transitions between locked and sliding, thus small differences in rupture speed will make a huge difference in slip over short periods.

The total amount of slip in this simulation ends up being several times larger than observed in the Tohoku-Oki earthquake (Simons et al., 2011), primarily because the fault continues to slip for quite some time after the rupture reaches the trench. We have made no attempt to adjust parameters to prevent this from occurring, as appears to be necessary to match observations. An important process that may contribute to cessation of slip is inelastic deformation of sedimentary material at shallow depths, which we have neglected here. This can be incorporated into future simulations using continuum plasticity, and we have already implemented this in our code (Dunham et al., in press). Furthermore, we have assumed a uniformly large initial stress, rather than a more realistic depth-dependent one.

In Figure 8 the vertical and horizontal displacement of the ocean floor is shown. The seafloor displacement is critical for tsunami prediction and hazards assess- 
(a) fully conservative method

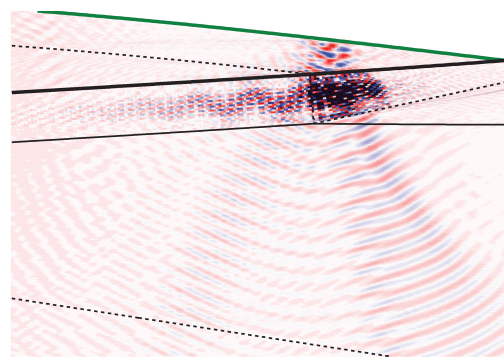

(b) stable method

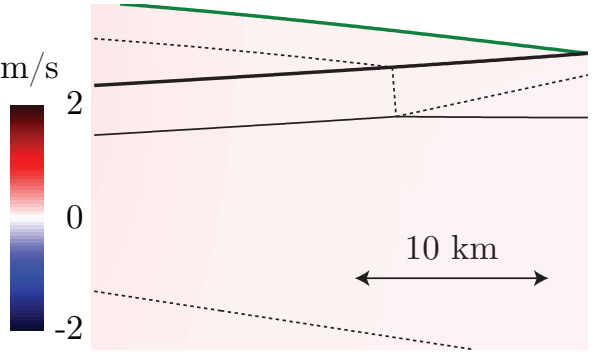

Fig. 9: Vertical particle velocity near the trench at $t=200 \mathrm{~s}$. (a) Shows the result when the provably stable method is used and (b) when the standard, fully conservative method is used. As can be seen the correction is critical for the long time integration on the highly skewed meshes that result from realistic geometries.

ment, as this is what causes the initial perturbation to the water column. Our model results are again several times larger than observed data from the TohokuOki earthquake (Sato et al., 2011), but this is to be expected given the unreasonably large amount of slip. The peak around $25 \mathrm{~km}$ is a transient feature associated with the passage of a Rayleigh wave propagating along the seafloor to the right. We again emphasize that we have made no attempt to modify initial conditions or friction law parameters to match observations. Furthermore, the solution may be sensitive to the location of the absorbing boundaries.

In order to assess the importance of energy stability, particularly for long time integration, Figure 9 compares the energy stable method with the non-energy stable, fully conservative $(\gamma=0)$ method. In particular, it shows the vertical particle velocity near the trench at $200 \mathrm{~s}$. Figure 9 (a) clearly shows that energy growth can occur when the conservative method is used. Such energy growth is not seen in Figure 9(b) for the simulation with the stability correction. The effects of this energy growth is not seen at earlier times as the growth rate (63) should be of order $\mathcal{O}\left(h^{p}\right)$.

\section{Conclusions}

We have developed a numerical method for simulation of earthquake ruptures in complex geometries. The method is based on the use of coordinate transforms and multi-block meshes to handle irregular domains. The method is based on the SBP-SAT formalism, and is provably stable with appropriately chosen penalty parameters.

Additionally, we have developed a correction term using a combination of the conservative and nonconservative form of the hyperbolic system which eliminates the additional energy growth in the numerical solution seen in previous work with coordinate transforms(Nordström and Carpenter, 2001; Nordström, 2006).

The accuracy and stability of the numerical method was verified using the method of manufactured solutions for a plane strain problem; the accuracy of the 
method for antiplane shear deformation was previously demonstrated by Kozdon et al. (2011).

A realistic application problem involving a subduction zone megathrust earthquake through multiple material layers demonstrated the versatility of the method for extremely challenging geometries. Additionally, it was shown that the energy growth due to using the standard, conservative formulation destroys the accuracy of the solution on the highly skewed meshes used for this problem, thus demonstrating the need for an energy stable method as developed in this paper.

\section{Acknowledgements}

J.E.K. and E.M.D were supported by National Science Foundation (NSF) award EAR-0910574 and the Southern California Earthquake Center (SCEC), as funded by NSF Cooperative Agreement EAR-0529922 and US Geological Survey Cooperative Agreement 07HQAG0008 (SCEC contribution number 1424). J.E.K. was also supported by NSF Fellowship for Transformative Computational Science using CyberInfrastructure OCI-1122734. The computations in this paper were conducted at the Stanford Center for Computational Earth and Environmental Science.

\section{A Proof of Proposition 1}

First, we note that normal stress and opening velocity can be written using the characteristic interface variables (26), (27), and (32)

$$
\begin{aligned}
\sigma_{n} & =\frac{1}{2}\left(\mathcal{W}_{P}^{-(1)}+w_{P}^{+(1)}\right)=-\frac{1}{2}\left(\mathcal{W}_{P}^{-(2)}+w_{P}^{+(2)}\right) \\
\beta & =\frac{1}{2 Z_{p}^{(1)}}\left(\mathcal{W}_{P}^{-(1)}-w_{P}^{+(1)}\right)-\frac{1}{2 Z_{p}^{(2)}}\left(\mathcal{W}_{P}^{-(2)}-w_{P}^{+(2)}\right) .
\end{aligned}
$$

Since the fault is closed, $\beta=0$, this implies that

$$
\left[\begin{array}{l}
\mathcal{W}_{P}^{-(1)} \\
\mathcal{W}_{P}^{-(2)}
\end{array}\right]=\frac{-1}{Z_{p}^{(1)}+Z_{p}^{(2)}}\left[\begin{array}{cc}
Z_{p}^{(1)}-Z_{p}^{(2)} & 2 Z_{p}^{(1)} \\
2 Z_{p}^{(2)} & Z_{p}^{(2)}-Z_{p}^{(1)}
\end{array}\right]\left[\begin{array}{l}
w_{P}^{+(1)} \\
w_{P}^{+(2)}
\end{array}\right]
$$

All that remains is to show that (38) can also be written in characteristic form (32). We can write the shear stress and slip velocity using the characteristic variables (26), and (27) as

$$
\begin{aligned}
\tau_{m}^{(l)} & =\frac{1}{2}\left(\mathcal{W}_{S V}^{-(l)}+w_{S V}^{+(l)}\right), \tau_{z}^{(l)}=\frac{1}{2}\left(\mathcal{W}_{S H}^{-(l)}+w_{S H}^{+(l)}\right) \\
V_{m} & =\frac{1}{2 Z_{s}^{(1)}}\left(\mathcal{W}_{S V}^{-(1)}-w_{S V}^{+(1)}\right)-\frac{1}{2 Z_{s}^{(2)}}\left(\mathcal{W}_{S V}^{-(2)}-w_{S V}^{+(2)}\right) \\
V_{z} & =\frac{1}{2 Z_{s}^{(1)}}\left(\mathcal{W}_{S H}^{-(1)}-w_{S H}^{+(1)}\right)-\frac{1}{2 Z_{s}^{(2)}}\left(\mathcal{W}_{S H}^{-(2)}-w_{S H}^{+(2)}\right) .
\end{aligned}
$$

The fault conditions (33) and (38) can then be written as the nonlinear system

$$
\mathbf{0}=\left[\begin{array}{c}
\tau_{m}^{(1)}+\tau_{m}^{(2)} \\
\tau_{z}^{(1)}+\tau_{z}^{(2)} \\
{\left[\begin{array}{c}
\tau_{m}^{(2)} \\
\tau_{z}^{(2)}
\end{array}\right]-\left[\begin{array}{c}
V_{m} \\
V_{z}
\end{array}\right] \frac{\sigma_{n} f(V, \psi)}{V}}
\end{array}\right], V=\sqrt{V_{m}^{2}+V_{z}^{2}} .
$$


The Jacobian of (120) with respect to the variables $\mathcal{W}_{S V}^{-(l)}$ and $\mathcal{W}_{S H}^{-(1)}$ is

$$
\begin{aligned}
\boldsymbol{J} & =\frac{1}{2}\left(\left[\begin{array}{cc}
\boldsymbol{I} & \boldsymbol{I} \\
\mathbf{0} & \boldsymbol{I}
\end{array}\right]+\left[\begin{array}{cc}
\mathbf{0} & \mathbf{0} \\
-\frac{1}{Z_{s}^{(1)}} \boldsymbol{J}_{F} & \frac{1}{Z_{s}^{(2)}} \boldsymbol{J}_{F}
\end{array}\right]\right), \\
\boldsymbol{J}_{F} & =\frac{\sigma}{V}\left(\left[\begin{array}{cc}
V_{m}^{2} & V_{m} V_{z} \\
V_{z} V_{m} & V_{z}^{2}
\end{array}\right] \frac{\frac{\partial f}{\partial V} V-f}{V^{2}}+f \boldsymbol{I}\right) .
\end{aligned}
$$

The determinant of $\boldsymbol{J}$ is

$$
J=\frac{1}{2 V \tilde{Z}_{s}}\left(V \tilde{Z}_{s}+2 \sigma_{n} f\right)\left(\tilde{Z}_{s}+2 \sigma_{n} \frac{\partial f}{\partial V}\right) .
$$

If the conditions of the proposition are satisfied then $J \neq 0$ and the proposition follows by the implicit function theorem.

\section{B Interpretation of the Scheme in the Original Variables}

For the north and south boundary and the fault the penalty vectors are of the form

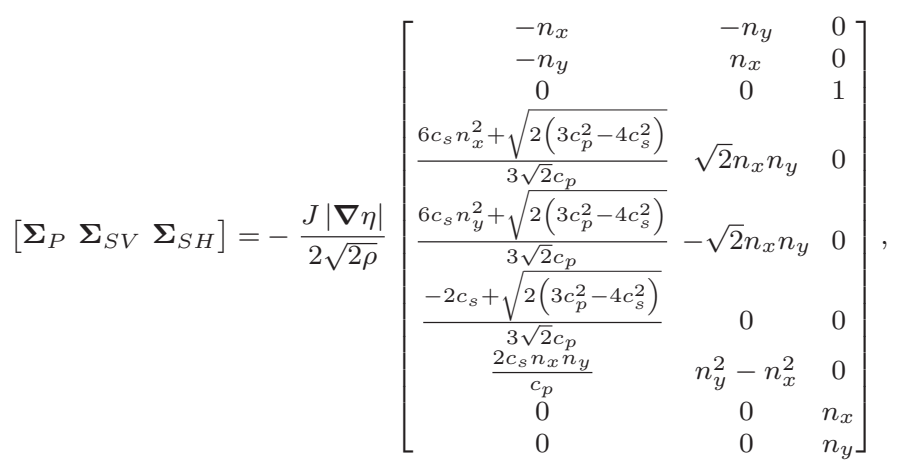

where, since we are only considering a single grid node on one side of the fault, we have dropped the superscript and subscript denoting these. For the east and west boundaries $|\nabla \eta|$ would be replaced by $|\nabla \xi|$.

If we transfer back to the original coordinate system the discrete governing equations become

$$
\begin{aligned}
\rho^{(l)} \boldsymbol{J}^{(l)} \frac{d \mathbf{v}_{i}^{(l)}}{d t}= & \boldsymbol{H}_{\xi}^{-1} \boldsymbol{Q}_{\xi} \boldsymbol{J}^{(l)} \frac{d \boldsymbol{\xi}^{(l)}}{d x_{j}} \boldsymbol{\sigma}_{i j}^{(l)}+\boldsymbol{H}_{\eta}^{-1} \boldsymbol{Q}_{\eta} \boldsymbol{J}^{(l)} \frac{d \boldsymbol{\eta}^{(l)}}{d x_{j}} \boldsymbol{\sigma}_{i j}^{(l)}+\mathbf{P} \mathbf{T}_{i}^{(l)} \\
\boldsymbol{J}^{(l)} \frac{d \boldsymbol{\sigma}_{i j}^{(l)}}{d t}= & \boldsymbol{H}_{\xi}^{-1} \boldsymbol{Q}_{\xi} \boldsymbol{J}^{(l)} \frac{d \boldsymbol{\xi}^{(l)}}{d x_{k}}\left[\delta_{i j} \lambda^{(l)} \mathbf{v}_{k}+G^{(l)}\left(\delta_{i k} \mathbf{v}_{i}+\delta_{j k} \mathbf{v}_{j}\right)\right] \\
& +\boldsymbol{H}_{\eta}^{-1} \boldsymbol{Q}_{\eta} \boldsymbol{J}^{(l)} \frac{d \boldsymbol{\eta}^{(l)}}{d x_{k}}\left[\delta_{i j} \lambda^{(l)} \mathbf{v}_{k}+G^{(l)}\left(\delta_{i k} \mathbf{v}_{i}+\delta_{j k} \mathbf{v}_{j}\right)\right]+\mathbf{P T}_{i j}^{(l)}
\end{aligned}
$$

where $\mathbf{P} \mathbf{T}_{i}^{(l)}$ and $\mathbf{P} \mathbf{T}_{i j}^{(l)}$ are the transformed penalty terms for the exterior boundaries and fault interface conditions. Considering the update for $v_{i}$ at a single grid node on the west boundary, the penalty term for this node (assuming it is not a corner node) is

$$
\begin{aligned}
P T_{i} & =-J\left[H_{\xi}\right]_{00} \frac{|\nabla \eta|}{2}\left[n_{i}\left(w_{P}^{-}-\mathcal{W}_{P}^{-}\right)+m_{i}\left(w_{S V}^{-}-\mathcal{W}_{S V}^{-}\right)+\hat{z}_{i}\left(w_{S H}^{-}-\mathcal{W}_{S H}^{-}\right)\right] \\
& =-n_{i} \frac{\rho}{T_{p}}\left(v_{P}-\hat{v}_{P}\right)-m_{i} \frac{\rho}{T_{s}}\left(v_{S V}-\hat{v}_{S V}\right)-\hat{z}_{i} \frac{\rho}{T_{s}}\left(v_{S H}-\hat{v}_{S H}\right),
\end{aligned}
$$


which shows that $v_{P}$ relaxes toward a target value of $\hat{v}_{P}$ over a time scale $T_{p}$. Similarly, $v_{S V}$ and $v_{S H}$ are relaxed to $\hat{v}_{S V}$ and $\hat{v}_{S H}$ over a time scale $T_{s}$. The target values are

$$
\hat{v}_{P}=\frac{\mathcal{W}_{P}^{-}-w_{P}^{+}}{2 Z_{p}}, \hat{v}_{S V}=\frac{\mathcal{W}_{S V}^{-}-w_{S V}^{+}}{2 Z_{s}}, \hat{v}_{S H}=\frac{\mathcal{W}_{S H}^{-}-w_{S H}^{+}}{2 Z_{s}},
$$

and the relaxation times are

$$
T_{p}=\frac{1}{J|\nabla \xi|} \frac{\left[\boldsymbol{H}_{\xi}\right]_{00}}{c_{p}}, \text { and } T_{s}=\frac{1}{J|\nabla \xi|} \frac{\left[\boldsymbol{H}_{\xi}\right]_{00}}{c_{s}}
$$

The prefactors $n_{i}, m_{i}$, and $\hat{z}_{i}$ weight the relative wave types influencing a particular component of velocity.

A similar calculation can be performed for the stresses at each boundary. For example, at the west boundary the penalty term for a non-corner node is

$$
\begin{aligned}
P T_{i j}= & -J\left[H_{\xi}\right]_{00} \frac{|\nabla \eta|}{2}\left\{c_{s}\left(n_{i} m_{i}+n_{j} m_{j}\right)\left(w_{S V}^{-}-\mathcal{W}_{S V}^{-}\right)\right. \\
& +c_{s}\left(n_{i} \hat{z}_{i}+n_{j} \hat{z}_{j}\right)\left(w_{S H}^{-}-\mathcal{W}_{S H}^{-}\right) \\
& \left.+c_{p}\left[\left(n_{i} n_{j}+m_{i} m_{j}+\hat{z}_{i} \hat{z}_{j}\right)-2 \frac{c_{s}^{2}}{c_{p}^{2}}\left(m_{i} m_{j}+\hat{z}_{i} \hat{z}_{j}\right)\right]\left(w_{P}^{-}-\mathcal{W}_{P}^{-}\right)\right\} \\
= & -\frac{1}{T_{s}}\left(n_{i} m_{i}+n_{j} m_{j}\right)\left(\sigma_{S V}-\hat{\sigma}_{S V}\right)-\frac{1}{T_{s}}\left(n_{i} \hat{z}_{i}+n_{j} \hat{z}_{j}\right)\left(\sigma_{S H}-\hat{\sigma}_{S H}\right) \\
& +\frac{1}{T_{P}} n_{i} n_{j}\left(\sigma_{n}-\hat{\sigma}_{n}\right)-\frac{1}{T_{P}} m_{i} m_{j}\left(\sigma_{m m}-\hat{\sigma}_{m m}\right)-\frac{1}{T_{P}} \hat{z}_{i} \hat{z}_{j}\left(\sigma_{z z}-\hat{\sigma}_{z z}\right)
\end{aligned}
$$

where $T_{P}$ and $T_{S}$ are defined in (129) and we have made the definitions

$$
\begin{aligned}
\hat{\sigma}_{n} & =-\frac{1}{2}\left(\mathcal{W}_{P}^{-}+w_{P}^{+}\right), \hat{\sigma}_{S V}=\frac{1}{2}\left(\mathcal{W}_{S V}^{-}+w_{S V}^{+}\right), \\
\hat{\sigma}_{S H} & =\frac{1}{2}\left(\mathcal{W}_{S H}^{-}+w_{S H}^{+}\right), \hat{\sigma}_{m m}=\stackrel{\leftrightarrow}{m}_{m}-\left(1-2 \frac{c_{s}^{2}}{c_{p}^{2}}\right) \hat{\sigma} \\
\hat{\sigma}_{z z} & =\stackrel{\leftrightarrow}{z}_{z}-\left(1-2 \frac{c_{s}^{2}}{c_{p}^{2}}\right) \hat{\sigma} .
\end{aligned}
$$

These expressions can be interpreted in a similar manner to the velocities.

Completely analogous expressions hold at the other boundaries and the fault since the penalty matrices are of a similar form.

\section{References}

B. T. Aagaard, T. H. Heaton, and J. F. Hall. Dynamic earthquake ruptures in the presence of lithostatic normal stresses: Implications for friction models and heat production. Bull. Seism. Soc. Am., 91(6):1765-1796, 2001. doi: 10.1785/0120000257.

J.-P. Ampuero. Etude physique et numérique de la nucléation des séismes. PhD thesis, Univ. Denis Diderot Paris, 2002.

D. J. Andrews. Rupture propagation with finite stress in antiplane strain. J. Geophys. Res., 81(20):3575-3582, 1976.

D. J. Andrews. Dynamic plane-strain shear rupture with a slip-weakening friction law calculated by a boundary integral method. Bull. Seism. Soc. Am., 75(1):1-21, 1985.

H. Aochi, E. Fukuyama, and M. Matsu'ura. Selectivity of spontaneous rupture propagation on a branched fault. Geophys. Res. Lett., 27(22):3635-3638, 2000.

D. Appelö and N. A. Petersson. A stable finite difference method for the elastic wave equation on complex geometrieswith free surfaces. Comm. Comp. Phys., 5(1):84-107, 2009. 
D. Appelö, T. Hagstrom, and G. Kreiss. Perfectly matched layers for hyperbolic systems: General formulation, well-posedness, and stability. SIAM J. Appl. Math., 67(1):1-23, 2006. doi: $10.1137 / 050639107$.

A. Bayliss, K. E. Jordan, B. J. Lemesurier, and E. Turkel. A fourth-order accurate finitedifference scheme for the computation of elastic waves. Bull. Seism. Soc. Am., 76(4):1115$1132,1986$.

M. H. Carpenter, D. Gottlieb, and S. Abarbanel. Time-stable boundary conditions for finitedifference schemes solving hyperbolic systems: Methodology and application to high-order compact schemes. J. Comp. Phys., 111(2):220-236, 1994. doi: 10.1006/jcph.1994.1057.

M. H. Carpenter, J. Nordström, and D. Gottlieb. A stable and conservative interface treatment of arbitrary spatial accuracy. J. Comp. Phys., 148(2):341-365, 1999.

M.H. Carpenter and C.A. Kennedy. Fourth-order 2N-storage Runge-Kutta schemes. Technical Report NASA TM-109112, National Aeronautics and Space Administration, Langley Research Center, Hampton, VA, 1994.

V. M. Cruz-Atienza and J. Virieux. Dynamic rupture simulation of non-planar faults with a finite-difference approach. Geophys. J. Int., 158(3):939-954, 2004. doi: 10.1111/j.1365246X.2004.02291.x.

S. Das. A numerical method for determination of source time functions for general threedimensional rupture propagation. Geophys. J. Roy. Astr. Soc., 62(3):591-604, 1980. doi: 10.1111/j.1365-246X.1980.tb02593.x.

S. Das and B. V. Kostrov. An investigation of the complexity of the earthquake source time function using dynamic faulting models. J. Geophys. Res., 93(B7):8035-8050, 1988.

S. M. Day. Three-dimensional finite difference simulation of fault dynamics: Rectangular faults with fixed rupture velocity. Bull. Seism. Soc. Am., 72(3):705-727, 1982.

S. M. Day, L. A. Dalguer, N. Lapusta, and Y. Liu. Comparison of finite difference and boundary integral solutions to three-dimensional spontaneous rupture. J. Geophys. Res., 110:B12307, 2005. doi: 10.1029/2005JB003813.

J. de la Puente, J.-P. Ampuero, and M. Käser. Dynamic rupture modeling on unstructured meshes using a discontinuous Galerkin method. J. Geophys. Res., 114:B10302, 2009. doi: 10.1029/2008JB006271.

J. H. Dieterich. Modeling of rock friction 1. Experimental results and constitutive equations. J. Geophys. Res., 84(B5):2161-2168, 1979. doi: 10.1029/JB084iB05p02161.

E. M. Dunham, D. Belanger, L. Cong, and J. E. Kozdon. Earthquake ruptures with strongly rate-weakening friction and off-fault plasticity: 1. Planar faults. Bull. Seism. Soc. Am., in press.

G. Festa and J-.P. Vilotte. The Newmark scheme as velocity and stress time-staggering: an efficient PML implementation for spectral element simulations of elastodynamics. Geophys. J. Int., 161(3):789-812, 2005. doi: 10.1111/j.1365-246X.2005.02601.x.

B. Fornberg. The pseudospectral method: Accurate representation of interfaces in elastic wave calculations. Geophys., 53(5):625-637, 1988. doi: 10.1190/1.1442497.

P. H. Geubelle and J. R. Rice. A spectral method for three-dimensional elastodynamic fracture problems. J. Mech. Phys. Solids, 43(11):1791-1824, 1995. doi: 10.1016/0022-5096(95)00043I.

B. Gustafsson. The convergence rate for difference approximations to mixed initial boundary value problems. Math. Comp., 29(130):396-406, 1975.

B. Gustafsson, H.-O. Kreiss, and J. Oliger. Time Dependent Problems and Difference Methods. Wiley-Interscience, 1996.

T. Hagstrom, A. Mar-Or, and D. Givoli. High-order local absorbing conditions for the wave equation: Extensions and improvements. J. Comput. Phys., 227(6):3322-3357, 2008. doi: 10.1016/j.jcp.2007.11.040.

N. Kame and T. Yamashita. Simulation of the spontaneous growth of a dynamic crack without constraints on the crack tip path. Geophys. J. Int., 139(2):345-358, 1999. doi: 10.1046/j.1365-246x.1999.00940.x.

Y. Kaneko, N. Lapusta, and J.-P. Ampuero. Spectral element modeling of spontaneous earthquake rupture on rate and state faults: Effect of velocity-strengthening friction at shallow depths. J. Geophys. Res., 113:B09317, 2008. doi: 10.1029/2007JB005553.

P. M. Knupp and S. Steinberg. The Fundamentals of Grid Generation. CRC Press, 1993.

J. E. Kozdon, E. M. Dunham, and J. Nordström. Interaction of waves with frictional interfaces using summation-by-parts difference operators: Weak enforcement of nonlinear boundary conditions. J. Sci. Comp, pages 1-27, 2011. doi: 10.1007/s10915-011-9485-3. 
H.-O. Kreiss. Initial boundary value problems for hyperbolic systems. Com. Pure Appl. Math., 23(3):277-298, 1970. doi: 10.1002/cpa.3160230304.

H.-O. Kreiss and G. Scherer. Finite element and finite difference methods for hyperbolic partial differential equations. In Mathematical aspects of finite elements in partial differential equations; Proceedings of the Symposium, pages 195-212, Madison, WI, 1974.

H.-O. Kreiss and G. Scherer. On the existence of energy estimates for difference approximations for hyperbolic systems. Technical report, Dept. of Scientific Computing, Uppsala University, 1977.

N. Lapusta, J. R. Rice, Y. Ben-Zion, and G. Zheng. Elastodynamic analysis for slow tectonic loading with spontaneous rupture episodes on faults with rate- and state-dependent friction. J. Geophys. Res., 105:23765-23790, 2000.

Y. Liu and J. Rice. Aseismic slip transients emerge spontaneously in three-dimensional rate and state modeling of subduction earthquake sequences. J. Geophys. Res., 110(B08307): 1-14, 2005. doi: 10.1029/2004JB003424.

S. Ma and P. Liu. Modeling of the perfectly matched layer absorbing boundaries and intrinsic attenuation in explicit finite-element methods. Bull. Seism. Soc. Am., 96(5):1779-1794, 2006. doi: $10.1785 / 0120050219$.

R. Madariaga, K. Olsen, and R. Archuleta. Modeling dynamic rupture in a 3D earthquake fault model. Bull. Seism. Soc. Am., 88(5):1182-1197, 1998.

L. E. Malvern. Introduction to the Mechanics of a Continuous Medium. Prentice Hall, 1st edition, 1977.

C Marone. Laboratory-derived friction laws and their application to seismic faulting. Annu. Rev. Earth Planet Sci., 26(1):643-696, 1998. doi: 10.1146/annurev.earth.26.1.643.

K. Mattsson and J. Nordström. Summation by parts operators for finite difference approximations of second derivatives. J. Comp. Phys., 199(2):503-540, 2004. doi: 10.1016/j.jcp.2004.03.001.

T. Miyatake. Numerical simulations of earthquake source process by a three- dimensional crack model. part I. rupture process. J. Phys. Earth, 28(6):565-598, 1980.

P. Moczo, J. Kristek, M. Gallis, P. Pazak, and M. Balazovjecha. The finite-difference and finite-element modeling of seismic wave propagation and earthquake motion. Acta Phys. Slovaca, 57(2):177-406, 2007.

S. Nilsson, N.A. Petersson, B. Sjogreen, and H.-O. Kreiss. Stable difference approximations for the elastic wave equation in second order formulation. SIAM J. Numer. Anal., 45(5): 1902-1936, 2007. doi: 10.1137/060663520.

H. Noda, E. M. Dunham, and J. R. Rice. Earthquake ruptures with thermal weakening and the operation of major faults at low overall stress levels. J. Geophys. Res., 114:B07302, 2009. doi: $10.1029 / 2008$ JB006143.

J. Nordström. Conservative finite difference formulations, variable coefficients, energy estimates and artificial dissipation. J. Sci. Comp., 29(3):375-404, 2006. doi: 10.1007/s10915005-9013-4.

J. Nordström and M. H. Carpenter. High-order finite difference methods, multidimensional linear problems, and curvilinear coordinates. J. Comp. Phys., 173(1):149-174, 2001. doi: 10.1006/jcph.2001.6864.

D. D. Oglesby, R. J. Archuleta, and S. B. Nielsen. Earthquakes on dipping faults: The effects of broken symmetry. Science, 280(5366):1055-1059, 1998. doi: 10.1126/science.280.5366.1055.

P. Olsson. Summation by parts, projections, and stability. II. Math. Comp., 64(212):1473$1493,1995$.

G. Perrin, J. R. Rice, and G. Zheng. Self-healing slip pulse on a frictional surface. J. Mech. Phys. Solids, 43(9):1461-1495, 1995. doi: 10.1016/0022-5096(95)00036-I.

J. R. Rice. Constitutive relations for fault slip and earthquake instabilities. Pure. Appl. Geophys., 121(3):443-475, 1983. doi: 10.1007/BF02590151.

J. R. Rice and A. L. Ruina. Stability of steady frictional slipping. J. Appl. Mech., 50(2): 343-349, 1983. doi: 10.1115/1.3167042.

J. R. Rice, N. Lapusta, and K. Ranjith. Rate and state dependent friction and the stability of sliding between elastically deformable solids. J. Mech. Phys. Solids, 49(9):1865-1898, 2001. doi: 10.1016/S0022-5096(01)00042-4.

P. J. Roache. Verification and Validation in Computational Science and Engineering. Albuquerque: Hermosa Publishers, 1998.

O. Rojas, E. M. Dunham, S. M. Day, L. A. Dalguer, and J. E. Castillo. Finite difference modelling of rupture propagation with strong velocity-weakening friction. Geophys. J. Int., 
179:1831-1858, 2009. doi: 10.1111/j.1365-246X.2009.04387.x.

A. Ruina. Slip instability and state variable friction laws. J. Geophys. Res., 88(B12): 10,359-10,370, 1983

M. Sato, T. Ishikawa, N. Ujihara, S. Yoshida, M. Fujita, M. Mochizuki, and Akira Asada. Displacement above the hypocenter of the 2011 Tohoku-Oki earthquake. Science, 332(6036): 1395, 2011. doi: 10.1126/science.1207401.

M. Simons, S. E. Minson, A. Sladen, F. Ortega, J. Jiang, S. E. Owen, L. Meng, J.-P. Ampuero, S. Wei, R. Chu, D. V. Helmberger, H. Kanamori, E. Hetland, A. W. Moore, and F. H. Webb. The 2011 magnitude 9.0 Tohoku-Oki earthquake: Mosaicking the megathrust from seconds to centuries. Science, 332(6036):1421-1425, 2011. doi: 10.1126/science.1206731.

W. S. Slaughter. The Linearized Theory of Elasticity. Birkhäuser, 2002.

B. Strand. Summation by parts for finite difference approximations for d/dx. J. Comp. Phys., 110(1):47-67, 1994. doi: 10.1006/jcph.1994.1005.

M. Svärd and J. Nordström. On the order of accuracy for difference approximations of initialboundary value problems. J. Comp. Phys., 218(1):333-352, 2007.

N. Takahashi, S. Kodaira, T. Tsuru, J.-O. Park, Y. Kaneda, K. Suyehiro, H. Kinoshita, S. Abe, M. Nishino, and R. Hino. Seismic structure and seismogenesis off Sanriku region, northeastern Japan. Geophys. J. Int., 159(1):129-145, 2004. doi: 10.1111/j.1365-246X.2004.02350.x.

E. Tessmer, D. Kosloff, and A. Behle. Elastic wave propagation simulation in the presence of surface topography. Geophys. J. Int., 108(2):621-632, 1992. doi: 10.1111/j.1365246X.1992.tb04641.x.

T. Tsuru, J.-O. Park, , N. Takahashi, S. Kodaira, Y. Kido, Y. Kaneda, and Y. Kono. Tectonic features of the Japan Trench convergent margin off Sanriku, northeastern Japan, revealed by multichannel seismic reflection data. J. Geophys. Res., 105(B7):16403-16413, 2000. doi: 10.1029/2000JB900132.

H. Zhang and X. Chen. Dynamic rupture process of the 1999 Chi-Chi, taiwan, earthquake. Ea. Sci., 22(1):3-12, 2009. doi: 10.1007/s11589-009-0003-8. 